Research Article

\title{
Determination of Electroacupuncture Effects on circRNAs in Plasma Exosomes in Diabetic Mice: An RNA-Sequencing Approach
}

\author{
Yin Shou $\mathbb{D}^{1},{ }^{1}$ Li Hu, ${ }^{2}$ Weibo Zhang, ${ }^{2}$ Yuan Gao, ${ }^{2}$ Ping Xu $\mathbb{D}^{\mathbb{D}},{ }^{2}$ and Bimeng Zhang $\mathbb{D}^{1}$ \\ ${ }^{1}$ Department of Acupuncture-Moxibustion, Shanghai General Hospital, Shanghai Jiao Tong University School of Medicine, \\ Shanghai 200080, China \\ ${ }^{2}$ Acumox and Tuina Research Section, College of Acumox and Tuina, Shanghai University of Traditional Chinese Medicine, \\ Shanghai 201203, China
}

Correspondence should be addressed to Ping Xu; xp99@163.com and Bimeng Zhang; acusci2007@126.com

Received 10 April 2019; Revised 5 July 2019; Accepted 29 July 2019; Published 24 September 2019

Academic Editor: Ghee T. Tan

Copyright (c) 2019 Yin Shou et al. This is an open access article distributed under the Creative Commons Attribution License, which permits unrestricted use, distribution, and reproduction in any medium, provided the original work is properly cited.

\begin{abstract}
circRNAs are involved in diabetes mellitus pathogenesis. Electroacupuncture (EA) is an effective therapeutic strategy for diabetes mellitus. However, whether the mechanism of action of EA on diabetes mellitus is related to altered circRNAs is unclear. The aim of this study was to reveal the effect of EA on circRNA expression in plasma exosomes and the underlying signaling pathway in mice with type 2 diabetes mellitus (T2DM). In total, 10 mice were randomly categorized into a normal group and 20 mice were used for the T2DM model preparation and randomly divided into the model and model + EA groups. Mice in the model + EA group were administered EA treatment. Changes in the fasting blood glucose (FBG) level and islet structure were evaluated. Plasma exosomes were subjected to RNA sequencing, and then bioinformatics analysis and real-time quantitative PCR (qPCR) verification were performed. EA treatment reduced the FBG level, preserved the islet structure, and reduced the islet $\beta$ cell apoptotic rate in T2DM mice. After EA treatment, 165 differentially expressed circRNAs were found. GO and KEGG analyses revealed that thyroid hormone signaling was actively regulated by EA. circRNA/miRNA interaction analysis revealed mmu-mir-7092-3p to be closely associated with circINPP4B, suggesting that the phosphatidylinositol signaling pathway may be affected by EA. qPCR confirmed that 12 circRNAs had significant differences. These findings suggested that EA intervention can significantly protect islet function and improve the FBG level in T2DM, possibly via regulation of thyroid hormone and phosphatidylinositol signaling.
\end{abstract}

\section{Introduction}

Diabetes mellitus is a chronic endocrine disorder caused by lifestyle changes or inadequate insulin secretion due to hereditary factors, islet dysfunction, or development of insulin resistance. It often has some serious complications such as diabetic nephropathy, diabetic ophthalmopathy, and pathological changes in the cardiovascular system and can endanger human life and health [1]. The International Diabetes Federation estimated that there were 451 million diabetic patients worldwide in 2017 and if no measures are taken, the number will increase to 642 million by 2040 [2]. At present, treatment of T2DM includes self-management, weight loss, dietary adjustment, exercise, and medicine. Drug therapy is effective but has many side effects [3-6]. Because of its tolerable and unstable efficacy, it is difficult to determine their optimum doses for maintaining healthy blood sugar levels and weight [7-9].

Electroacupuncture (EA) is an integral part of traditional Chinese medicine and has been used to treat human illnesses for at least the past 3000 years. Acupuncture is reported to promote weight loss $[10,11]$. In a statement issued by the American Diabetes Association (ADA) in 2005, acupuncture therapy was suggested to be effective for neuralgia at any stage of type 2 diabetes [12,13]. In a single-blind experiment, acupuncture was found to significantly alleviate diabetic 
gastroparesis [14]. Acupuncture is also effective in treating diabetes mellitus with myasthenia gravis [15] and that with lower extremity arterial disease [16]. Recent experimental studies have shown that acupuncture can treat T2DM by regulating insulin resistance, improving islet beta cell function, and alleviating endothelial dysfunction [17, 18], which may involve AMPK and P13K/Akt mTORC1 signaling [19-21].

Exosomes, extracellular nanovesicles $(30-150 \mathrm{~nm})$ of endocytic origin, have been found to transport many biological molecules such as DNA fragments, circRNAs, and micro(mi)RNAs to promote intercellular communication and have also been shown to regulate many pathophysiological processes including immune response, inflammation, and infection [22]. Among these biological molecules transported by exosomes, the role of circRNAs, which are noncoding RNAs that function as miRNA “sponges" in various diseases, in diabetes mellitus has gradually attracted scientists' attention in recent years. A previous study showed that 529 circRNAs were aberrantly expressed in diabetic retinopathy and were involved in diabetic retina pathogenesis [23]. Another study revealed that circHIPK3 played a role in diabetic retinopathy by blocking miR-30a function, causing increased endothelial proliferation and vascular dysfunction [24]. Additionally, it was reported that 247 circRNAs were dysregulated in T2DM patients with depression [25]. Recently, it was pointed out that reduced expression of hsa_circ_0056891, hsa_circ_0063425, and hsa_circ_0071336 is an independent predictor of T2DM and increased the risk of T2DM [26]. Although there have been a few reports about relation between circRNAs and diabetes mellitus, whether the mechanism of action of acupuncture on diabetes mellitus is related to altered circRNAs remain unexplored.

In this study, for the first time, we investigated the effects of EA on circRNA expression in plasma exosomes and the signaling pathway of T2DM in vivo. Our results may provide new insights for elucidating the mechanism of EA in treating diabetes mellitus.

\section{Materials and Methods}

2.1. Experimental Animals. In total, 30 male C57BL/6 mice weighing $18 \pm 2 \mathrm{~g}$ were obtained from the Animal Laboratory Center, First People's Hospital Affiliated to Shanghai Jiaotong University. All mice were maintained in the Animal Laboratory Center of First People's Hospital Affiliated to Shanghai Jiaotong University in a controlled environment of $25 \pm 2^{\circ} \mathrm{C}$, relative humidity $55-70 \%$, and 12-h light/dark cycle. The mice were housed 5/cage, with free access to food and water. All experimental methods were approved by the Animal Ethics Committee of the First People's Hospital Affiliated to Shanghai Jiaotong University.

2.2. Animal Model. All male C57BL/6 mice were fed with basic forage (purchased from the Animal Breeding Center of Shanghai First People's Hospital) for one week for adaptation. Then, 10 mice were categorized according to their body weight into the normal $(\mathrm{N})$ group (fed with basic forage). The remaining 20 mice were prepared as the T2DM model by feeding with high-forage diets consisting of casein $22.8 \%$, dextrin $17 \%$, DL-methionine $0.2 \%$, minerals $4 \%$, sodium bicarbonate $10.5 \%$, vitamins $1 \%$, heavy tartaric acid choline $0.2 \%$, sucrose $17.5 \%$, soybean oil $2.5 \%$, hydrogenated coconut oil $33.35 \%$, and potassium citrate $0.4 \%$. After 6 weeks, these 20 mice were intraperitoneally injected with $1 \%$ streptozotocin (STZ, Sigma-Aldrich) solution (STZ dissolved in sodium citrate buffer) at a dose of $100 \mathrm{mg} / \mathrm{kg}$ daily for 7 consecutive days. For one week after the injection, fasting blood glucose (FBG) level and randomized blood glucose level was monitored. The model was considered unsuccessful if the randomized blood glucose level was less than $16.8 \mathrm{mmol} / \mathrm{L}$ or if the FBG level was less than $11.1 \mathrm{mmol} / \mathrm{L}$. STZ was then injected at a dose of $150 \mathrm{mg} / \mathrm{kg}$ for the second time. The T2DM model mice were randomized into two groups: model group and model + EA group.

2.3. EA Intervention. After routine disinfection of acupoints, acupuncture Zusanli and Pishu points were stimulated on the mice in the model + EA group using needles with a diameter of $0.22 \mathrm{~mm}$ and length of $13 \mathrm{~mm}$. After insertion of the needles, EA (frequency $2 \mathrm{~Hz}$, intensity $1 \mathrm{~mA}-3 \mathrm{~mA}$, Intermittent waveform, serial length $30 \mathrm{~s}$, increasing once every 5 minutes, lasting for 15 minutes) was applied on the acupoints by connecting with a low-frequency pulse therapy instrument (G6805-2, Shanghai Medical Device High-tech Company, China), with slight shaking in the local muscle. The intervention was applied once every two days, 3 times a week, for 4 weeks, and 12 times in total.

2.4. FBG Measurement. FBG was measured before and after the modelling and after EA intervention for two weeks. After 16 hours of fasting, blood samples were collected from the tail vein of mice and applied to the test strip (lot: 4174080, Johnson \& Johnson), with the test strip in the Johnson blood glucose meter. After 5 seconds, blood glucose values were read and recorded. To prevent infection, aureomycin eye ointment (purchased from Huaqing Pharmaceutical Company, Xinxiang, China) was applied to the tail wound on the day after testing.

2.5. Preparation of Pancreas Slices. At the end of the experiment, mice were anesthetized by intraperitoneal injection with pentobarbital sodium $(60 \mathrm{mg} / \mathrm{kg})$, and the whole blood of mice was extracted quickly with a $1 \mathrm{ml}$ sterile syringe. The pancreas was quickly removed from the mice and washed with normal saline, dried with filter paper, fixed in $4 \%$ polyformaldehyde for 24 hours, embedded in paraffin, and sectioned for hematoxylin-eosin staining [27] and for the TDT-mediated dUTP-biotin nick end-labeling (TUNEL) to examine the apoptosis rate in islet beta cells [28]. The 
tissue and cell structure was observed under an optical microscope, and photographs were taken.

2.6. Extraction of Plasma Exosomes. Exosomes from blood plasma were isolated by differential centrifugation. Briefly, blood plasma $(1 \mathrm{ml})$ was diluted with $6 \mathrm{ml}$ PBS and differentially centrifuged at $1900 \times g$ for $10 \mathrm{~min}, 3000 \times g$ for $15 \mathrm{~min}, 500 \times \mathrm{g}$ for $10 \mathrm{~min}$, and $20000 \times g$ for $20 \mathrm{~min}$ at $4^{\circ} \mathrm{C}$ to eliminate cell debris, followed by centrifugation at $100000 \times g$ for $70 \mathrm{~min}$. The resulting pellet was resuspended in $1 \mathrm{ml}$ PBS, followed by centrifugation at $100000 \times \mathrm{g}$ for $70 \mathrm{~min}$ at $4^{\circ} \mathrm{C}$. The supernatant was removed, and the resulting exosomal pellet was reconstituted in $30 \mu \mathrm{L}$ of PBS and stored at $-80^{\circ} \mathrm{C}$.

2.7. Western Blotting. Exosomes were further characterized by western blotting. Protein concentration of exosomes was quantified using a BCA protein assay kit (Beyotime, China). The protein samples were separated on an $8 \%$ SDS-PAGE gel and transferred to polyvinyl difluoride (PVDF) membranes using a Mini Trans-Blot cell transfer apparatus (Bio-Rad, USA). After blocking with 5\% BSA for $1 \mathrm{~h}$, the membranes were washed 3 times ( 5 min each time) with $1 \mathrm{x}$ TBST and probed overnight with antibodies against CD63 (1:1000, Abcam), CD9 (1:2000, Abcam, USA), TSG101 $(1: 3000$, Santa Cruz Biotechnology, USA), and calnexin $(1: 1000$, Cell Signaling Technology, USA) at $4^{\circ} \mathrm{C}$. The primary antibody was then discarded, and the membrane was washed 3 times (5 min each time) with $1 \mathrm{x}$ TBST and incubated with $1: 2000$ diluted secondary antibody at room temperature for $1 \mathrm{~h}$ and washed thrice with $1 \mathrm{x}$ TBST for $10 \mathrm{~min}$ each. The membrane was then covered with enhanced chemiluminescence (BOSTER) substrate and imaged in a gel imaging system (Bio-Rad).

2.8. Transmission Electron Microscopy. Transmission electron microscopy was used to visualize the morphological characteristics of exosomes. In total, $3 \mu \mathrm{L}$ of sample $(1 \mathrm{mg} / \mathrm{ml})$ was added onto a 300 mesh Formvar-coated copper grid and was fixed under an infrared lamp for 30 minutes. Samples not adhering to the copper grids were carefully removed with filter paper and then incubated in $2.5 \%$ glutaraldehyde phosphate buffer for 15 minutes. After rinsing with PBS and distilled water for 2 times, respectively, the samples were negatively stained with phosphotungstic acid and visualized using a Philips CM120 transmission electron microscope (Philips, Amsterdam, Holland) operated at $80 \mathrm{kV}$.

2.9. Nanoparticle Tracking Analysis. Exosomes were diluted and subjected to nanoparticle-tracking analysis (NTA) using a ZetaView PMX 110 (PMX, Germany) at $15000.00 \mathrm{US} / \mathrm{cm}$ conductivity, pH 7.4 electrolyte, and $27.34^{\circ} \mathrm{C}$. The particle trajectory was recorded and the concentration and diameter distribution of the sample were output. The exosome concentration of the original solution was obtained according to the dilution.
2.10. Exosomal RNA Isolation. For RNA extraction, $20 \mu \mathrm{L}$ of exosome suspension was mixed with $1 \mathrm{ml}$ Trizol buffer and placed on ice for 5 minutes. Then, $200 \mu \mathrm{L}$ of chloroform was added and the mixture placed on ice for 15 minutes after vigorous shaking for 30 seconds, followed by centrifugation at $13500 \times \mathrm{g}$ for 10 minutes at $4^{\circ} \mathrm{C}$. After the upper layer was transferred to a new enzyme-free tube, precooled isopropanol was added to this layer and mixed, and the mixture was kept at room temperature for 10 minutes. After centrifugation of this mixture at $13500 \times g$ for 10 minutes at $4^{\circ} \mathrm{C}$, the supernatant was discarded and washed twice with $75 \%$ ethanol. The ethanol was then removed and the pellets were air dried for 15 minutes. Finally, $24 \mu \mathrm{L}$ of RNase-free water was added to the extracted RNA and stored at $-80^{\circ} \mathrm{C}$. The quantity and quality of the RNA were determined using the NanoDrop ND-1000 (Thermo Fisher Scientific, Waltham, MA, USA) and Agilent Bioanalyzer 2100 (Agilent Technologies, Santa Clara, CA, USA).

2.11. RNA Library Preparation and Sequencing. The rRNAs in total RNA were removed using Ribo-Zero rRNA Removal Kits (Illumina, USA). Then, TruSeqStranded Total RNA Library Prep Kit (Illumina, USA) was used to preprocess the RNA and construct the sequencing library. The quantity and quality of the RNA were determined using Agilent Bioanalyzer 2100 (Agilent Technologies, Santa Clara, CA, USA). According to the Illumina sequencing instructions, the $10 \mathrm{pM}$. library was denatured as a single-stranded DNA molecule, captured in an Illumina flowcell, amplified into clusters in situ, and sequenced over 150 cycles on an Illumina HiSeq sequencer using a two-terminal mode. The library was constructed and sequenced by Shanghai Yunxu Biotechnology Co., Ltd.

2.12. Annotation of Host Linear Transcripts and Identification of Differentially Expressed circRNAs. After sequencing on the Illumina HiSeq 4000 sequencer, paired-end reads were retrieved. Cut adapt [29] (v1.9.3) software was used to eliminate the low-quality reads and obtain high-quality reads. STAR software (v2.5.1b) [30] was used to compare the high-quality reads to the reference genome/transcriptome, and DCC software (v0.4.4) [31] was used to detect and identify circRNA. The identified circRNA was annotated based on the circBase database [32]. circRNAs with fold changes $\geq 2.0$ or fold change $\leq 0.5$ in expression level in the model + EA group compared with both the normal and model group identified as differentially expressed. Linear transcripts were annotated according to the location of the chromosome where the circRNA sequence was overlapped.

2.13. GO and KEGG Pathway Analysis of Linear Transcripts. To analyze the potential functions of linear transcripts, the identified linear transcripts sequences were mapped with Gene Ontology Terms (http://geneontology.org/). GO term matching was performed with blast2go and go2protein. Gene functions were classified into three subgroups, namely, biological process, cellular components, and molecular 
function. To analyze the statistical significance of GO terms, we employed hypergeometric tests. The top 10 enriched GO terms affected by EA ranked by enrichment score were presented.

To annotate the identified differential linear transcripts in each pathway, Kyoto Encyclopedia of Genes and Genomes (KEGG) pathway (http://www.genome.jp/kegg/ pathway.html) analysis was conducted. To analyze the statistical significance of KEGG pathway enrichment, we employed hypergeometric tests. The top 10 pathway enrichment of upregulated and downregulated differentially expressed host linear transcripts affected by EA intervention were presented.

2.14. miRNA Target Prediction. In order to explore the function of circRNAs, putative interactions between differentially expressed circRNAs and their target miRNAs were theoretically evaluated using TargetScan and miRanda database. A hit between any expressed miRNA (including the new predicted miRNA) and a target circRNA was considered for a miRanda score of 140 or higher.

2.15. circRNA-miRNA Coexpression Network Analysis. Evidences have shown that circRNAs could bind with miRNAs and function as natural miRNA sponges to influence related miRNAs' activities. circRNA-miRNA coexpression network was built based on the prediction of miRNA-binding sites and the correlations between circRNA and miRNA. Two downregulated and three upregulated circRNAs were selected to generate a network map with cytoscape software (V. 3.2.1). Yellow nodes represented circRNAs and green nodes represented miRNAs.

2.16. Hierarchical Clustering Analysis. To generate an overview of differentially expressed circRNA profiles among the three groups, hierarchical clustering analysis was conducted based on the expression values of all target circRNAs and differentially expressed circRNAs using the Cluster and TreeView programs.

2.17. Quantitative Real-Time PCR. To validate the RNA sequencing data, we performed qPCR analysis of 12 differentially expressed genes. cDNA reverse transcription was performed from total RNA using the iScript Reverse Transcriptase Kit (Biorad, Hercules, CA). GAPDH was selected as a reference gene for all experiments. All primers for qPCR were synthesized by Sangon Biotech, China. The primer sequences used for $\mathrm{qPCR}$ are detailed in Supplementary Table S1. qPCR was performed using the SYBR Green Supermix kit (Bio-Rad) per the manufacturer's instructions. After activation of the polymerase enzyme at $95^{\circ} \mathrm{C}$ for $10 \mathrm{~min}, 40 \mathrm{cycles}$ of $95^{\circ} \mathrm{C}$ for $10 \mathrm{~s}, 60^{\circ} \mathrm{C}$ for $60 \mathrm{~s}$, and $95^{\circ} \mathrm{C}$ for $15 \mathrm{~s}$ were performed on the Gene Amp PCR System 9700 (Applied Biosystems, Foster City, CA). Melting curve analysis was used to confirm the specificity of the amplification reactions. Relative gene expression was quantified in triplicate $(n=3)$. The relative expression levels were calculated using the $2^{-\Delta \Delta C T}$ method.

2.18. Statistical Analysis. All data were processed using SPSS 19.0 software. Normal distribution data are expressed as mean \pm SD and were analyzed using ANOVA. The skewed distribution data are expressed as median $(M)$ and quartile spacing $(Q 1, Q 2)$, and the rank sum test was used for intergroup comparison. $P<0.05$ was considered significant.

\section{Results and Discussion}

3.1. General Information on Mice. There were 8 mice with random blood sugar level higher than $16.8 \mathrm{mmol} / \mathrm{L}$ and 1 with fasting blood sugar level higher than $11.1 \mathrm{mmol} / \mathrm{L}$; and the success rate of the model was $45 \%$. The remaining 11 mice were injected with STZ for a second time, with a $100 \%$ success rate. After high-fat feeding, the body shape of the mice changed, revealing central obesity and sparse hair. After STZ injection, the mice became depressed, showed decreased activities, had dry and sparse fur, excreted decreased urine volume, and consumed higher volumes of drinking water; all changes were significant.

3.2. Effect of EA Intervention on FBG Level. After modelling, the FBG levels in mice of the M group and model + EA group were markedly increased. After 4 weeks of EA intervention, the FBG levels of the $M$ group and model + EA group were still remarkably higher than that of the $\mathrm{N}$ group $(P \leq 0.001)$ (Table 1). However, a significant reduction in FBG level was observed in the model + EA group when compared with that in the $M$ group (Table 1). EA was thus suggested to significantly improve the FBG level although it could not restore the normal FBG level.

\subsection{Effect of EA on Pancreatic Tissue and Islet $\beta$ Cell Apoptosis} Rate. The pancreas is an important organ for secreting insulin and regulating the blood sugar levels. In order to study the effect of EA on pancreatic tissue in T2DM, hematoxylin-eosin staining was performed. As shown in Figure 1(a), the pancreas of normal mice showed intact islet structure, regular arrangement of pancreatic cells, and clear nuclei. In the $\mathrm{M}$ group, a large number of inflammatory cells infiltrated, surface adipocytes degenerated, interstitial hyperplasia, vasodilation, congestion, and necrosis were observed in the pancreatic tissue, with no complete islet structure (Figure 1(b)). In the model + EA group, mild and moderate inflammatory cell infiltration, vasodilation, and hyperemia were observed in the pancreatic tissue of mice and the structure of islets was preserved in the sections (Figure 1(c)). These results indicated that EA could effectively protect the pancreatic tissue in T2DM and inhibit the effect of STZ.

Further TUNEL analysis showed that the apoptosis rate of islet $\beta$ cells in the model and model + EA groups was obviously higher than that in the $\mathrm{N}$ group (Table 2). Nevertheless, the apoptosis rate of islet $\beta$ cells in the $M+E A$ 
TABLE 1: EA's intervention effect on FBG $[(\bar{X} \pm \mathrm{SD}) ; M(Q 1, Q 2)]$.

\begin{tabular}{lcccc}
\hline Groups & $(n)$ & \multicolumn{3}{c}{ FBG (mmol/L) } \\
& & Before modelling & After modelling & 14 th day of EA treatment \\
\hline Model group & 10 & $8.96 \pm 1.11$ & $14.27 \pm 2.17^{*}$ & $22.88(20.85,24.91)^{* *}$ \\
Model + EA group & 10 & $8.76 \pm 1.17$ & $14.77 \pm 2.40^{*}$ & $15.80(14.09,17.51)^{\Delta *}$ \\
Normal group & 10 & $8.51 \pm 1.21$ & $9.45 \pm 1.10$ & $9.54(8.70,10.37)^{\Delta *}$ \\
\hline
\end{tabular}

Note. ${ }^{*, \Delta, \#}$ Statistical differences $(P<0.001)$ compared with the normal group, model group, and model + EA group, respectively.

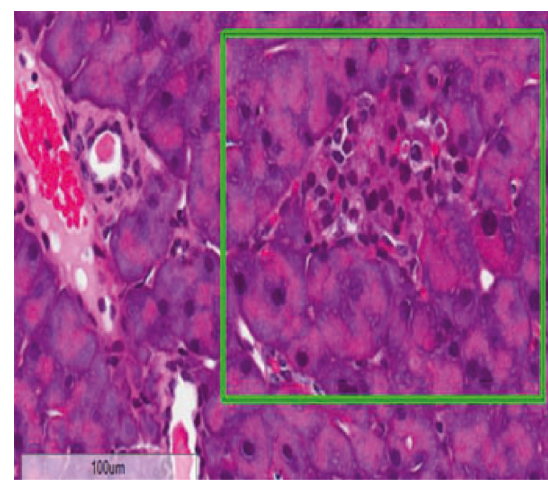

(a)

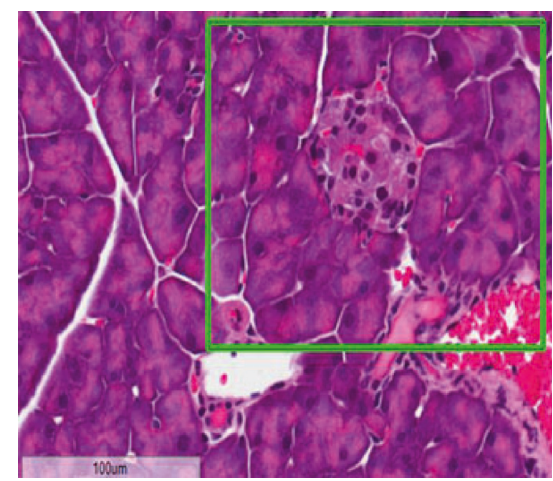

(b)

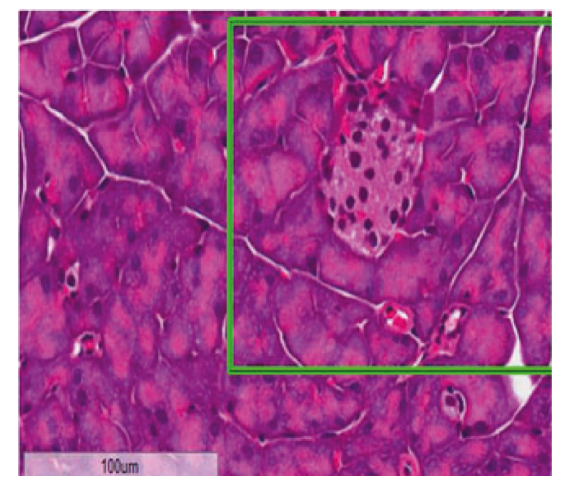

(c)

FIgURE 1: Hematoxylin-eosin staining in the pancreatic tissue of the model group (a), model + EA group (b), and normal group (c). Magnification: 400x. Islet structure is marked with a green box.

TABLE 2: EA's intervention effect on the apoptosis rate of islet $\beta$ cell $[M(Q 1, Q 2)]$.

\begin{tabular}{lcc}
\hline Groups & $(n)$ & Apoptosis rate $(\%)$ \\
\hline Model group & 15 & $9.77(3.48,20.11)^{* \#}$ \\
Model + EA group & 15 & $5.31(2.80,8.63)^{\Delta^{*}}$ \\
Normal group & 15 & $2.24(0.00,5.73)^{\Delta \#}$ \\
\hline
\end{tabular}

Note. ${ }^{*, \Delta, \# \text { Statistical differences }(P<0.05, P<0.01 \text {, and } P<0.01 \text {, re- }}$ spectively) compared with the normal group, model group, and model $+\mathrm{EA}$ group, respectively.

group was still significantly lower than that in the M group, implying that EA could significantly improve the apoptotic rate of islet $\beta$ cells.

\subsection{Identification of Differentially Expressed circRNA Profiles} in Plasma Exosomes. Plasma exosomes confirmed by western blotting, transmission electron microscopy, and NTA (Supplementary Figure S1) were subjected to RNA sequencing. As a result, a total of 579 circRNA targets were found in the plasma exosomes of three groups (Supplementary Table S2). To provide a comprehensive landscape of the origination of circRNAs, linear transcripts of circRNAs from the corresponding genes were annotated and the circRNAs distribution in the genome was also explored according to the location of the chromosome where the circRNA sequence was overlapped (Supplementary Table S2). Differentially expressed circRNAs were displayed through fold change filtering (Figure 2(a)-2(c), Supplementary Table S3). Additionally, 165 circRNAs were detected to be differentially expressed in the $\mathrm{M}+\mathrm{EA}$ group compared with the $\mathrm{M}$ and $\mathrm{N}$ groups. The distribution of the differentially expressed
circRNAs in the $\mathrm{M}+\mathrm{EA}$ group on the mouse chromosomes is shown in Figure 2(d). Among these, 21 circRNAs, which were downregulated in the $M$ group when compared with the $\mathrm{N}$ group, were upregulated after EA treatment, whereas 144 circRNAs that were upregulated in the $M$ group compared to the $\mathrm{N}$ group were downregulated after EA treatment. The top ten upregulated and downregulated circRNAs are listed in Table 3 by fold change. Hierarchical clustering analysis indicated that these differentially expressed circRNA expression pattern were distinguishable among three groups (Figure 3 ).

3.5. GO Annotation and Pathway Enrichment Analysis. Under the assumption that circRNA function would be related to the known function of the host linear transcripts, differentially regulated linear transcripts were further mapped with Gene Ontology Terms (http://geneontology. org/). According to GO annotations, all identified differentially expressed circRNAs among the three groups were divided into three categories: cellular components, biological processes, and molecular functions (Supplementary Figure S2, Supplementary Figure S3). We employed a hypergeometric test, followed by FDR (false discovery rate) multiple correction to calculate the $P$ value of each GO item for enrichment analysis. In total, GO items of differentially host linear transcripts affected by EA were involved in basic metabolism, cell metabolism, cell macromolecule metabolism, organic substance metabolism, cell growth, development, and other related functions (Figure 4). The top 10 GO annotations enriched for the upregulated and downregulated linear transcripts are displayed in Tables 4 and 5, respectively. 


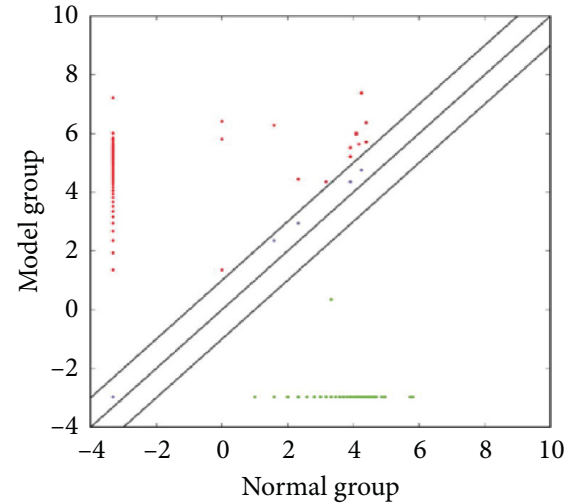

(a)

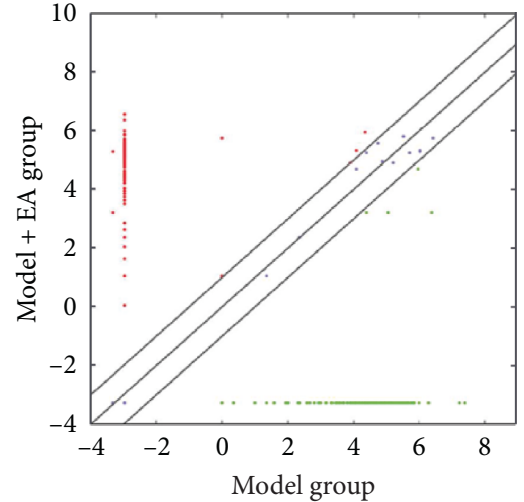

(b)

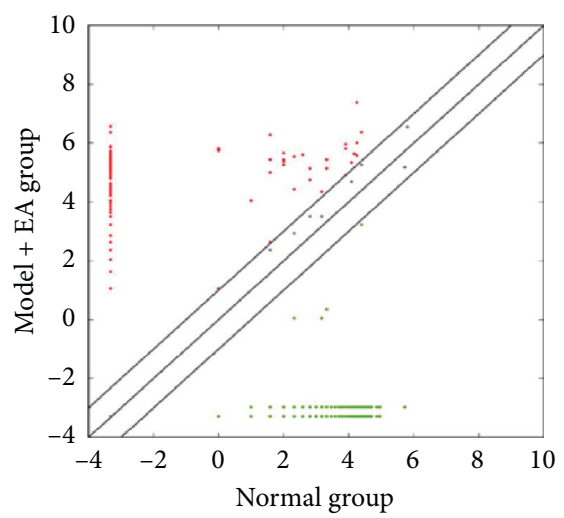

(c)

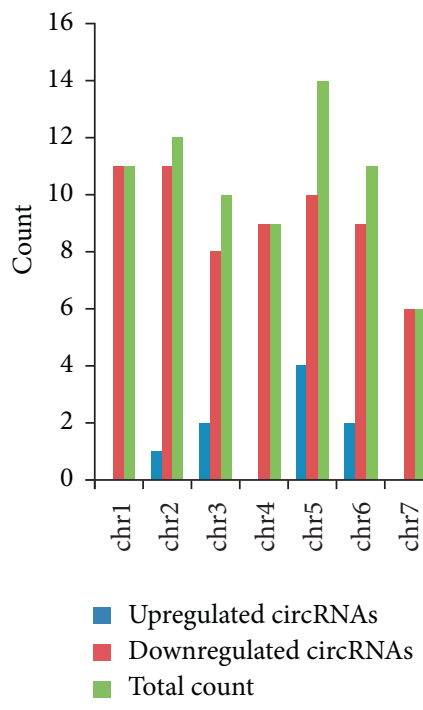

(d)

FIGURE 2: Differences and characterizations of circRNA expression profiles in plasma exosomes among the three groups. (a) Scatter plots between model and normal groups. (b) Scatter plots between model and model + EA groups. (c) Scatter plots between model + EA and normal groups. The values plotted on $X$ and $Y$ axes of scatter plots are the averaged normalized signal values of each group (log 2 scaled). The middle line refers to no difference between the two groups. The circRNAs above the middle line and below the middle line indicate more than 2.0-fold changes between two groups. (d) The distribution of differentially expressed circRNAs in the model + EA group in mouse chromosomes.

To obtain an overview of the main biochemical metabolic pathways, all differentially expressed host linear transcripts were analyzed using KEGG, to provide an alternative functional annotation of genes according to their related biochemical pathways (Supplementary Table S4). Our analysis revealed that 165 differentially expressed host linear transcripts affected by EA treatment in T2DM were involved in a total of 56 annotated pathways (Figure 5). The upregulated host linear transcripts mainly belonged to the following pathways: phospholipase D signaling pathway, thyroid hormone signaling pathway, oxytocin signaling pathway, cancer choline metabolic pathway, platelet activation, cell cycle, phosphatidylinositol signaling system, $B$ cell receptor signaling pathway, lysine degradation, and African trypanosomiasis. The top 10 results of upregulated pathway enrichment affected by EA treatment are listed in Table 6. On the contrary, the downregulated host linear transcripts mainly belonged to the following pathways: $\mathrm{F} c \gamma \mathrm{R}$ mediates the macrophage phagocytosis signal transduction pathway, HIF-1 signal transduction pathway, phosphatidylinositol signal system pathway, cancer choline metabolism pathway, lysine degradation pathway, NOD-like receptor signaling pathway, mTOR signaling pathway, phosphoinositol (IP) metabolism pathway, and cancer proteoglycan pathway. The top 10 results of pathway enrichment affected by EA treatment are listed in Table 7 .

3.6. Prediction of circRNA/miRNA Interaction and Construction of an Interaction Network. circRNAs act as a "microRNA sponge" to fine-tune the levels of microRNAs. In order to explore the function of circRNAs, interactions between differentially expressed circRNAs and their target miRNAs were theoretically predicted by microRNA target 
TABLE 3: The top 10 upregulated and downregulated circRNAs ranked by fold changes.

\begin{tabular}{|c|c|c|c|}
\hline circRNA & Circ ID & circRNA type & Fold change \\
\hline \multicolumn{4}{|l|}{ Upregulated circRNAs } \\
\hline chr11:103046379-103055289+ & mmu_circ_0000325 & Exonic & 746.5258 \\
\hline chr6:47576536-47577667- & mmu_circ_0001471 & Exonic & 738.4114 \\
\hline chr10:40076564-40080807- & 10_39800613_39796370_-4243|20.91|10|17 & Exonic & 649.1528 \\
\hline chr9:64160821-64161441- & 9_64009248_64008628_-620|14.59|13|40 & Exonic & 503.0934 \\
\hline chr7:115659516-115662444- & 7_122805958_122803030_-2928|21.29|10|16 & Exonic & 462.5214 \\
\hline chr11:72767580-72767898+ & Novel & Sense overlapping & 454.4070 \\
\hline chr4:84956276-84979521+ & 4_84625425_84602180_-23245|17.55|11|14 & Exonic & 413.8349 \\
\hline chr2:5052988-5054716- & 2_4975762_4974034_-1728|15.56|5|6 & Exonic & 397.6061 \\
\hline chr12:91289489-91299077- & 12_92537517_92527929_-9588|12.77|4|6 & Exonic & 381.3773 \\
\hline chr4:138146285-138151828+ & Novel & Exonic & 373.2629 \\
\hline \multicolumn{4}{|l|}{ Downregulated circRNAs } \\
\hline chr6:119920110-119921028+ & mmu_circ_0001516 & Exonic & 1492.2043 \\
\hline chr16:32950292-32961744+ & mmu_circ_0000681 & Exonic & 637.69416 \\
\hline chr13:55213204-55238862+ & 13_55340223_55314565_-25658|19.14|4|4 & exonic & 573.92475 \\
\hline chr13:103884143-103897928- & 13_104688008_104674223_-13785|17.98|20|70 & Sense overlapping & 561.17086 \\
\hline chr16:76330746-76352549- & mmu_circ_0000702 & Exonic & 548.41698 \\
\hline chr12:51619814-51661713- & mmu_circ_0000370 & Exonic & 535.66310 \\
\hline chr11:61736916-61745060- & Novel & Exonic & 510.15533 \\
\hline chr17:24607437-24619570- & Novel & exonic & 497.40145 \\
\hline chr1:165911288-165913349- & 1_167843480_167841419_-2061|15.43|10|28 & Exonic & 484.64756 \\
\hline chr12:35078900-35086984+ & 12_35771656_35763572_-8084|19.56|22|64 & Exonic & 471.89368 \\
\hline
\end{tabular}

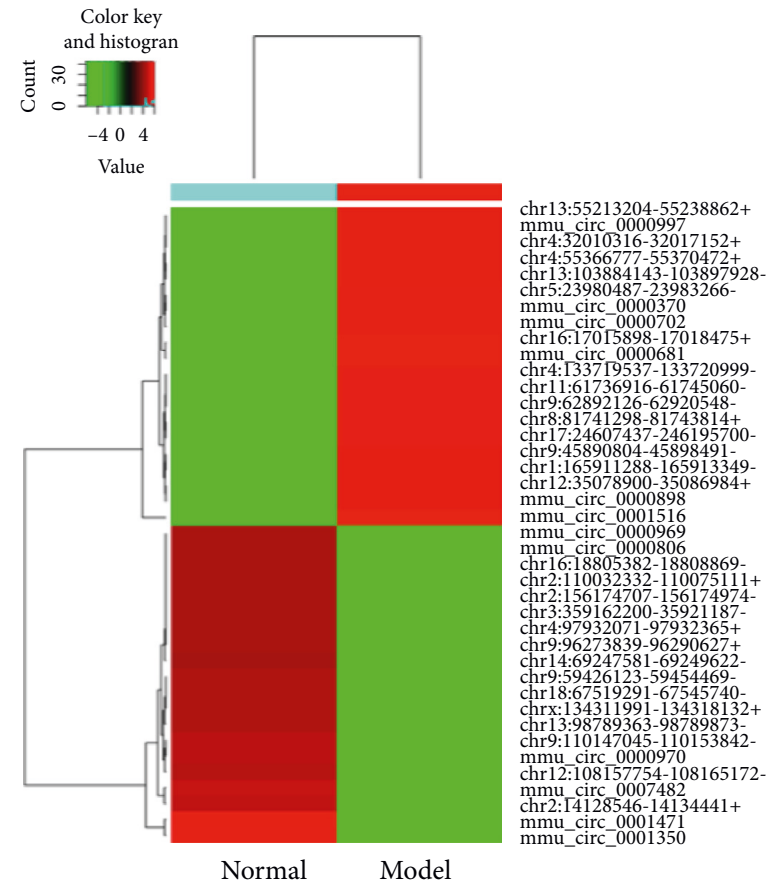

(a)

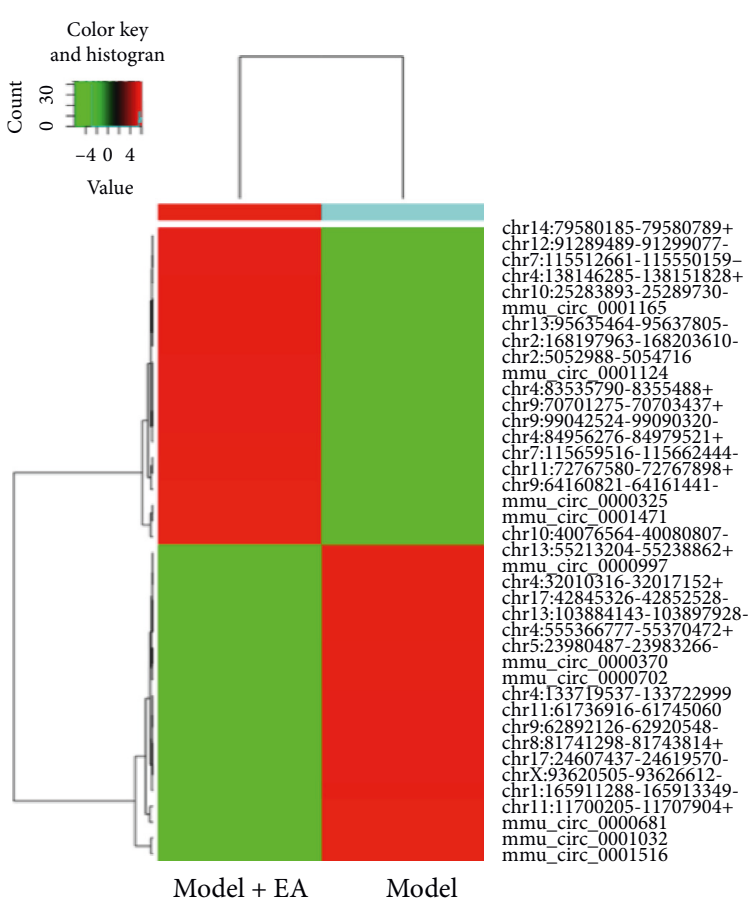

(b)

Figure 3: Continued. 


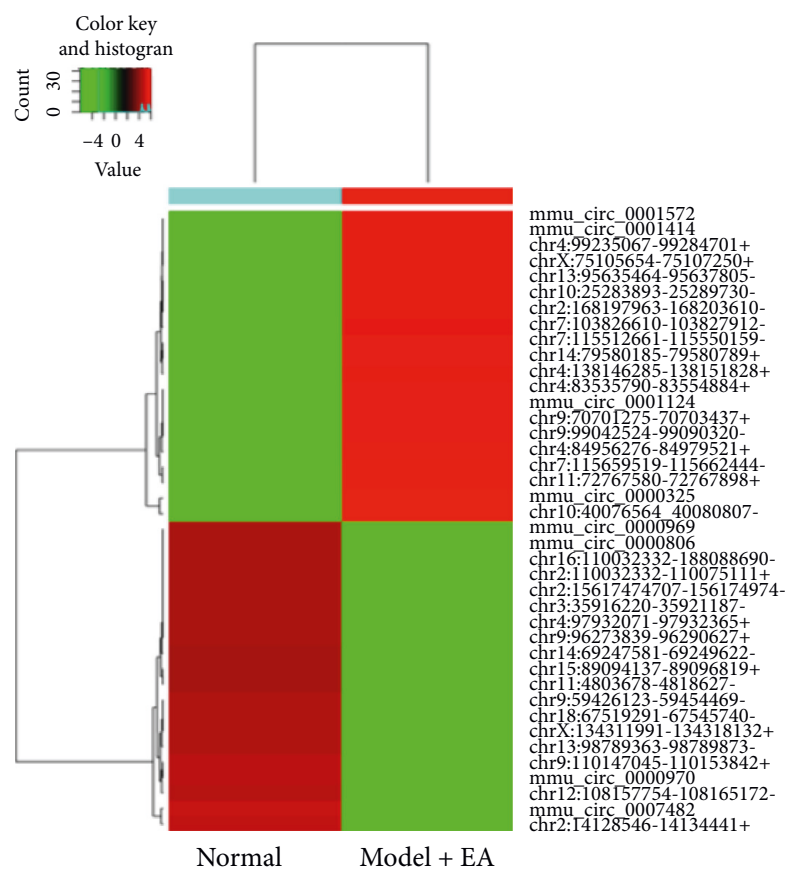

(c)

FIGURE 3: Heat map and hierarchical clustering showing expression values of all differentially expressed circRNAs among the three groups. Each column represents a sample and each row represents a circRNA. Red strip represents high relative expression and green strip represents low relative expression. (a) Hierarchical cluster analysis between model and normal groups. (b) Hierarchical cluster analysis between model and model + EA groups. (c) Hierarchical cluster analysis between normal and model + EA groups.

gene prediction software according to the TargetScan and miRanda database. Supplementary Table S5 displays each circRNA and its potential complementary binding miRNAs. The predicted expression of top 10 upregulated circRNAbound microRNAs and downregulated circRNA-bound microRNAs affected by EA are shown in Tables 8 and 9 . Furthermore, an entire interaction network of two downregulated (mmu-circ000550 and mmu-circ001018) and three upregulated circRNAs (mmu_circ_0001124, mmucirc006255, and mmu-circ006982) with their acting miRNAs were delineated using Cytoscape (Figure 6).

3.7. Validation of the Differentially Expressed circRNAs. Twelve differentially expressed circRNA genes, namely, circPwwp2a, circPde5a, circEzh2, circTlk1, circBtaf1, circHipk2, circBrd4, circCep128, circStrn3, circPrrc2b, circZwilch, and circTulp4, were randomly selected and verified by qRTPCR. The $\log 2$ fold changes were calculated for RNA-seq data and qPCR results (Figure 7(a) and 7(b)). Most qRT-PCR results matched well with the RNA-seq data.

\section{Discussion}

It is well known that insulin resistance and beta cell dysfunction are the main pathogenic factors of T2DM. Previous studies have shown that EA can treat T2DM by regulating insulin resistance and improving the function of islet beta cells. The majority of acupoints selected by EA are Zusanli, Pishu, Weiwanxia Yu, and Zhongwan, among others
$[17,18]$. In this study, the Zusanli and Pishu points were selected for EA application, which resulted in an obvious decrease in FBG levels in the T2DM model mice. These results indicated that EA had a certain therapeutic effect on relieving hyperglycemia, which was consistent with the results of previous studies [17, 33, 34].

Some studies have been conducted with regard to the potential pathways by which EA exerts its therapeutic effect on diabetes mellitus. A previous study by Tominaga et al. [21] showed that repeated application of EA to the Zusanli is capable of improving diet-induced insulin resistance, probably through activation of AMPK signaling in skeletal muscles. Lan et al. [19] found that EA mitigates endothelial dysfunction via effects on the PI3K/Akt signaling pathway in high-fat-diet-induced insulin-resistant rats. On the contrary, Leng et al. [20] found that EA can improve obesity and reduce the potential risk of type 2 diabetes via hypothalamic Tscl promoter demethylation and by inhibiting the activity of mTORC1 signaling pathway. These results are in accordance with our results. We also found that EA treatment could upregulate the signaling pathways of thyroid hormone, sphingolipid biosynthesis (ganglion series), cGMPPKG, cancer transcriptional error regulation, cancer choline metabolism, Rap1, actin cytoskeleton regulation, and adipocyte lipid regulation and could downregulate the $\mathrm{F} c \gamma \mathrm{R}$ mediated macrophage phagocytosis signal transduction pathway, HIF-1 signal pathway, phosphatidylinositol signal system pathway, cancer choline metabolism pathway, lysine degradation pathway, NOD-like receptor signal pathway, mTOR signal pathway, phosphoinositol (IP) metabolism 


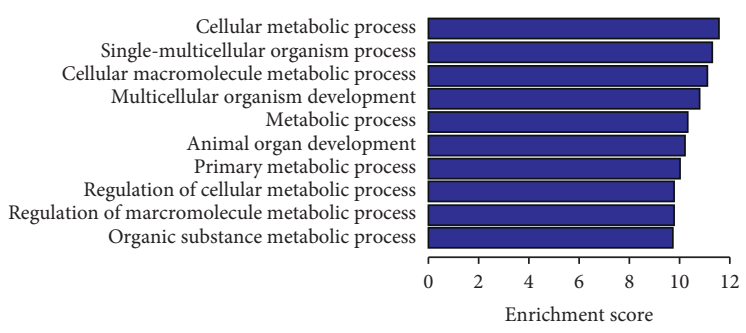

(a)

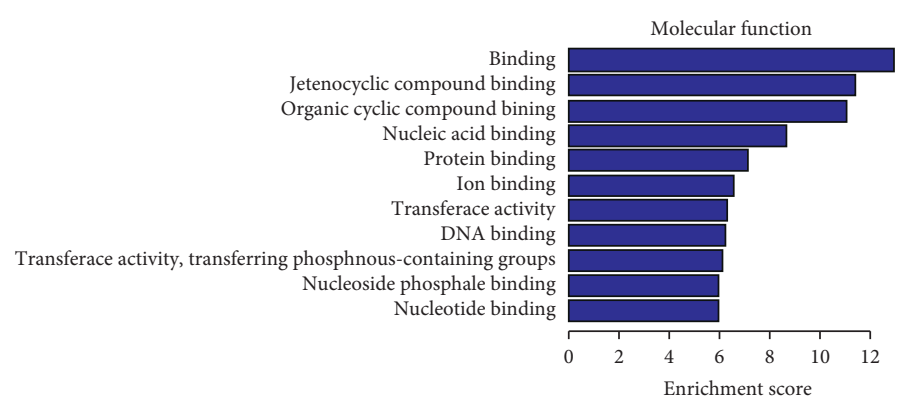

(c)

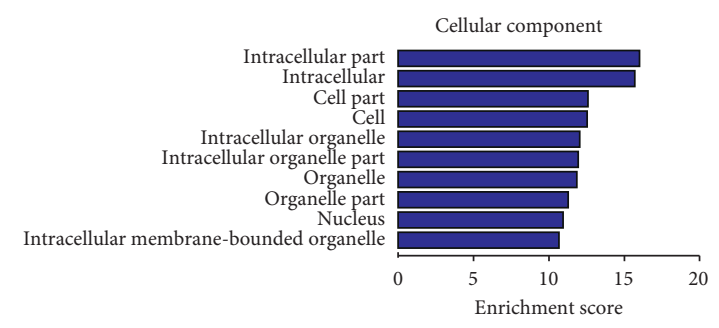

(e)

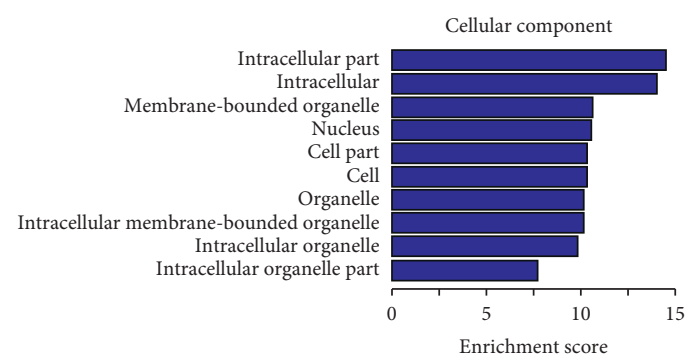

(b)

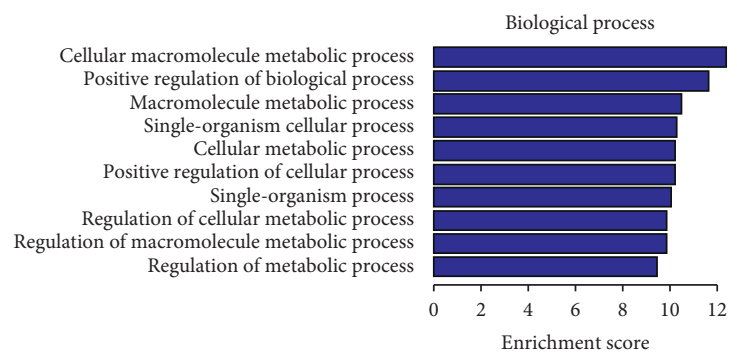

(d)

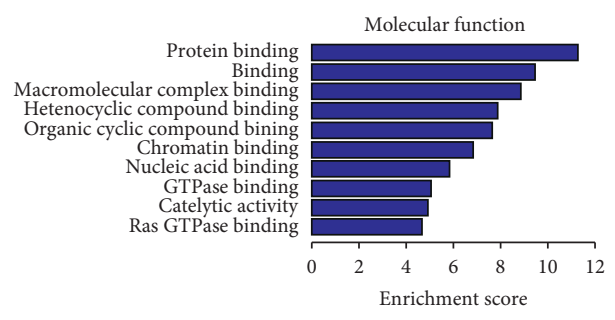

(f)

Figure 4: Gene ontology (GO) annotation of host linear transcripts affected by EA from the Gene Ontology Terms database (http://www. geneontology.org) compared to the normal ( $a, b, c)$ and model group (d, e, f). (a, d) Cellular components; (b, e) biological process; (c, f) molecular function.

TABLE 4: The top $10 \mathrm{GO}$ annotations enrichment of upregulated differentially expressed host linear transcripts affected by EA intervention.

\begin{tabular}{|c|c|c|c|c|}
\hline Gene function & $\begin{array}{c}\text { Number of } \\
\text { genes }\end{array}$ & $P$ & $\begin{array}{l}\text { False discovery } \\
\text { rate }\end{array}$ & Differentially expressed genes \\
\hline Cellular macromolecule metabolic process & 73 & $<0.05$ & $<0.05$ & $\begin{array}{l}\text { CNPT1, ARID1A, STRN3, DKC1, GAB1, } \\
\text { EZH2, MBD2, NCOA2, SOX6, ZEB1, etc. }\end{array}$ \\
\hline Positive regulation of biological process & 59 & $<0.05$ & $<0.05$ & $\begin{array}{l}\text { GAB1, EIF4G3, TGFBR2, PDE5A, ADCY9, } \\
\text { ASPH, SULF1, CNOT1, CTCF, DNMT1, etc. }\end{array}$ \\
\hline Macromolecule metabolic process & 74 & $<0.05$ & $<0.05$ & $\begin{array}{l}\text { BRD4, CTCF, DNMT1, ETV6, EZH1, EZH2, } \\
\text { HECTD1, UBE3A, KLHL7, KIF16B, etc. }\end{array}$ \\
\hline Single-organism cellular process & 93 & $<0.05$ & $<0.05$ & $\begin{array}{l}\text { CTCF, ZFP207, BRD4, ANP32B, GAB1, IARS, } \\
\text { HECTD1, CNOT1, CLIC4, SULF1, etc. } \\
\text { MED13L, ACAACA, SDHC, ADCY9, ASCC3, }\end{array}$ \\
\hline Cellular metabolic process & 78 & $<0.05$ & $<0.05$ & $\begin{array}{l}\text { STK39, RABGEF1, EIF4G3, ELF1, PLAGL1, } \\
\text { etc. }\end{array}$ \\
\hline Positive regulation of cellular process & 53 & $<0.05$ & $<0.05$ & $\begin{array}{l}\text { GAB1, EIF4G3, TGFBR2, ADCY9, Adam10, } \\
\text { ADNP, UBE3A, CTCELF1, SOX6, etc. }\end{array}$ \\
\hline Single-organism process & 98 & $<0.05$ & $<0.05$ & $\begin{array}{c}\text { OPTN, CTCF, ZFP207, BRD4, ANP32B, } \\
\text { CIGALT1, UBE3A, TGFBR2, ST6GALNAC3, } \\
\text { ST3GAL5, etc. }\end{array}$ \\
\hline Regulation of cellular metabolic process & 57 & $<0.05$ & $<0.05$ & $\begin{array}{l}\text { EIF4G3, RABGEF1, STK39, OPTN, KIF16B, } \\
\text { RERE, ELF2, MLLT3, ATAD2, GPBP1, etc. }\end{array}$ \\
\hline $\begin{array}{l}\text { Regulation of macromolecule metabolic } \\
\text { process }\end{array}$ & 57 & $<0.05$ & $<0.05$ & $\begin{array}{l}\text { AGO2L, ELF1, ADNP, ARID4B, EIF4G3, } \\
\text { TGFBR2, PLAGL1, CD46, PIK3CB, UBE2K, } \\
\text { etc. }\end{array}$ \\
\hline Regulation of metabolic process & 60 & $<0.05$ & $<0.05$ & $\begin{array}{l}\text { BRD41, CTCF, DNMT1, ETV6, EZH1, EZH2, } \\
\text { ATAD2B, PDGFD, ERBB2IP, DKC1, etc. }\end{array}$ \\
\hline
\end{tabular}


TABLE 5: The top $10 \mathrm{GO}$ annotations enrichment of downregulated differentially expressed host linear transcripts affected by EA intervention.

\begin{tabular}{|c|c|c|c|c|}
\hline Gene function & $\begin{array}{l}\text { Number of } \\
\text { genes }\end{array}$ & $P$ & $\begin{array}{l}\text { False discovery } \\
\text { rate }\end{array}$ & Differentially expressed genes \\
\hline Cellular metabolic process & 90 & $<0.05$ & $<0.05$ & $\begin{array}{l}\text { POLA1, WHSC1, HIPK2, HOXB3, NFKB1, } \\
\text { RAD23B, RAD52, FBXO18, ST3GAL6, PLCG2, } \\
\text { etc. }\end{array}$ \\
\hline Single-multicellular organism process & 69 & $<0.05$ & $<0.05$ & $\begin{array}{c}\text { CBFB, SP3, PRKCA, MAP3K7, NRIP1, } \\
\text { TNRC6C, IKZF1, NFKB1, NEK1, LATS2, etc. }\end{array}$ \\
\hline Cellular macromolecule metabolic process & 79 & $<0.05$ & $<0.05$ & $\begin{array}{l}\text { POLA1, WHSC1, HIPK2, HOXB3, NFKB1, } \\
\text { PIAS1, ZBTB20, PRDM5, PHF14, EHMT1, etc. }\end{array}$ \\
\hline Multicellular organism development & 61 & $<0.05$ & $<0.05$ & $\begin{array}{l}\text { PRKCA, MAP3K7, NRIP1, CHM, ATF6, } \\
\text { NEK1, LATS2, TSC2, IFT57, ARID11A, etc. } \\
\text { CBFB, ARIH2, UBE2D2A, ANKIB1, TRIP12, }\end{array}$ \\
\hline Metabolic process & 95 & $<0.05$ & $<0.05$ & $\begin{array}{l}\text { ARID1A, STRN3, MAP3K7, PRKCA, DUSP3, } \\
\text { etc. }\end{array}$ \\
\hline Animal organ development & 47 & $<0.05$ & $<0.05$ & $\begin{array}{l}\text { NRIP1, ATF6, SP3, IKZF1, NEK1, PLCG2, } \\
\text { WHSC2, WHSC1, ZFPM1, MATR3, etc. } \\
\text { PICALM, PRPSAP2, PIP5K1B, MECR, PLCG2, }\end{array}$ \\
\hline Primary metabolic process & 88 & $<0.05$ & $<0.05$ & $\begin{array}{l}\text { CD2AP, EP400, MLLT10, TTC7B, FAM126A, } \\
\text { etc. }\end{array}$ \\
\hline Regulation of cellular metabolic process & 63 & $<0.05$ & $<0.05$ & $\begin{array}{c}\text { ZMYM5, EFEMP1, SP3, POU2F1, AFF3, } \\
\text { IFT57, GON4L、TNRC6C, LARP4B, } \\
\text { RPS6KB1, etc. }\end{array}$ \\
\hline $\begin{array}{l}\text { Regulation of macromolecule metabolic } \\
\text { process }\end{array}$ & 63 & $<0.05$ & $<0.05$ & $\begin{array}{l}\text { TCEA1, PLCG2, FBX018, RBM4, DUSP3, } \\
\text { PRKCA, BOLL, TSC2, MATR3, PTPN22, etc. }\end{array}$ \\
\hline Organic substance metabolic process & 90 & $<0.05$ & $<0.05$ & $\begin{array}{l}\text { ARIH2, UBE2D2A, ANKIB1, TRIP12, STRN3, } \\
\text { RSRC11, CDK13, SRBD1, ERI3, CCNE1, etc. }\end{array}$ \\
\hline
\end{tabular}

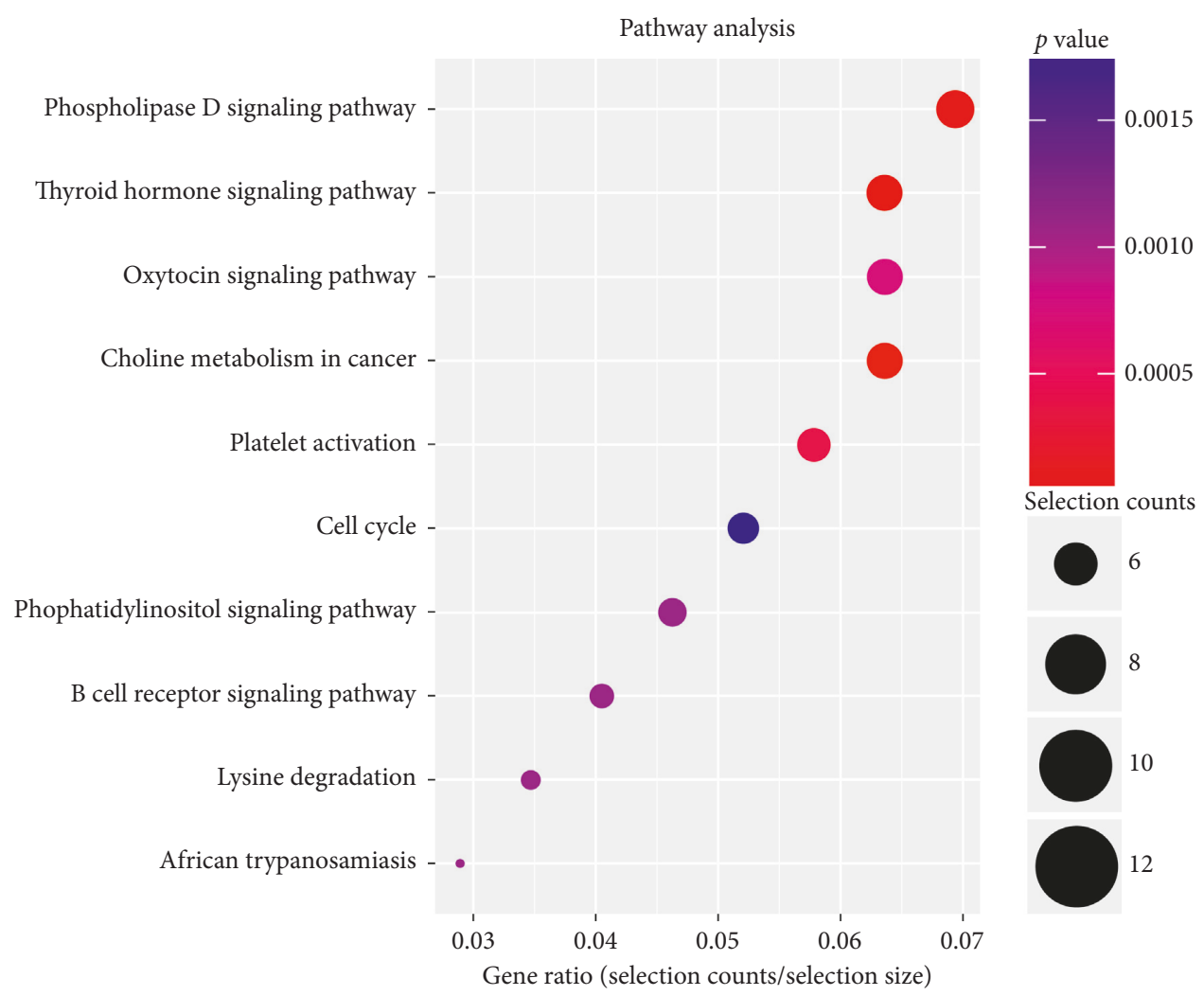

Figure 5: KEGG pathway enrichment of host linear transcripts affected by EA. The dot plot shows the gene ratio value of the top ten most significant enrichment pathways. 
TABLE 6: The top 10 pathway enrichment of upregulated differentially expressed host linear transcripts affected by EA intervention.

\begin{tabular}{lcccc}
\hline Pathway & $\begin{array}{c}\text { Selection } \\
\text { counts }\end{array}$ & $P$ & $\begin{array}{c}\text { False discovery } \\
\text { rate }\end{array}$ & Differentially expressed genes \\
\hline Thyroid hormone signaling pathway & 5 & $<0.05$ & $<0.05$ & MED13, MED13L, NCOA2, PIK3CB, SLC16A10 \\
Glycosphingolipid biosynthesis-ganglio series & 2 & $<0.05$ & $<0.05$ & ST3GAL5, ST6GALNAC3 \\
cGMP-PKG signaling pathway & 4 & $<0.05$ & $<0.05$ & ADCY9, PDE5A, PIK3CB, ROCK1 \\
Transcriptional misregulation in cancer & 4 & $<0.05$ & $<0.05$ & ETV6, MLLT3, TGFBR2, ZEB1 \\
Choline metabolism in cancer & 3 & $<0.05$ & $<0.05$ & PCYT1A, PDGFD, PIK3CB \\
Rap1 signaling pathway & 4 & $<0.05$ & $<0.05$ & ADCY9, FYB, PDGFD, PIK3CB \\
Regulation of actin cytoskeleton & 4 & $<0.05$ & $<0.05$ & IQGAP2, PDGFD, PIK3CB, ROCK1 \\
AMPK signaling pathway & 3 & $<0.05$ & $<0.05$ & ACACA, PIK3CB, RAB14 \\
Platelet activation & 3 & $<0.05$ & $<0.05$ & ADCY9, PIK3CB, ROCK1 \\
Regulation of lipolysis in adipocytes & 2 & $<0.05$ & $<0.05$ & ADCY9, PIK3CB \\
\hline
\end{tabular}

TABLE 7: The top 10 pathway enrichment of downregulated differentially expressed host linear transcripts affected by EA intervention.

\begin{tabular}{lcccc}
\hline Pathway & Selection counts & $P$ & False discovery rate & Differentially expressed genes \\
\hline Fc gamma R-mediated phagocytosis & 5 & $<0.05$ & $<0.05$ & PIP5K1B, PLCG2, PRKCA, RPS6KB1, CIN \\
HIF-1 signaling pathway & 5 & $<0.05$ & $<0.05$ & NFKB1, PDK1, PLCG2, PRKCA, RPS6KB1 \\
Phosphatidylinositol signaling system & 4 & $<0.05$ & $<0.05$ & INPP4B, PIP5K1B, PLCG2, PRKCA \\
Choline metabolism in cancer & 4 & $<0.05$ & $<0.05$ & PIP5K1B, PRKCA, RPS6KB1, TSC2 \\
Lysine degradation & 3 & $<0.05$ & $<0.05$ & EHMT1, NSD1, WHSC1 \\
NOD-like receptor signaling pathway & 3 & $<0.05$ & $<0.05$ & ERBB2IP, MAP3K7, NFKB1 \\
Thyroid hormone signaling pathway & 4 & $<0.05$ & $<0.05$ & MED12L, PLCG2, PRKCA, TSC2 \\
mTOR signaling pathway & 3 & $<0.05$ & $<0.05$ & PRKCA, RPS6KB1, TSC2 \\
Inositol phosphate metabolism & 3 & $<0.05$ & $<0.05$ & INPP4B, PIP5K1B, PLCG2 \\
Proteoglycans in cancer & 5 & $<0.05$ & $<0.05$ & CTTN, KB1 \\
\hline
\end{tabular}

TABLE 8: The predicted expression of top 10 upregulated circRNA-bound microRNAs affected by EA.

\begin{tabular}{|c|c|c|c|c|c|}
\hline $\operatorname{circR}$ & $2 \mathrm{NA}$ & A & A & miRNA & $\mathrm{miR}$ \\
\hline $\begin{array}{l}\text { chr5 } \\
1185\end{array}$ & $\begin{array}{l}\mathrm{mmu}-\mathrm{miR}-7028- \\
5 \mathrm{p}\end{array}$ & $\mathrm{mm}$ & $-5 p$ & $5 \mathrm{p}$ & ul-miR \\
\hline chr6:72128264 & $\begin{array}{c}\mathrm{mmu}-\mathrm{miR}-7669- \\
3 \mathrm{p}\end{array}$ & amu-miR & $\mathrm{mmu}-\mathrm{m}$ & mu-mil & $\mathrm{nmu}-\mathrm{miR}$ \\
\hline hr3: & mmu-miR-6 & $\mathrm{mn}$ & $\mathrm{m}$ & $5 p$ & $\begin{array}{c}\text { mmu-miR-103-2- } \\
5 \mathrm{p}\end{array}$ \\
\hline chr10:40076 & $\begin{array}{c}\mathrm{mmu}-\mathrm{miR}-6954- \\
5 \mathrm{p}\end{array}$ & $\mathrm{mm}$ & iiR-1966-5p & mmu-miR-708-5p & mmu-miR-7013-5p \\
\hline chr11:8 & $\mathrm{mml}$ & $\begin{array}{c}\text { mmu-miR-7 } \\
5 \mathrm{p}\end{array}$ & p & $3 \mathrm{p}$ & $3 p$ \\
\hline chr9:99042 & $\begin{array}{l}\text { mmu-miR-7032- } \\
5 \mathrm{p}\end{array}$ & $\underset{5 p}{m m u-m i R-3069-}$ & $\begin{array}{r}\mathrm{mmu}-\mathrm{mi} \\
5\end{array}$ & $\begin{array}{r}\text { mmu-miR- } \\
5 p\end{array}$ & $\mathrm{mmu}-\mathrm{miR}$ \\
\hline $\operatorname{chr} 16: 42855$ & mmu-miR-204-3p & mmu-miR-191-3p & mmu-miR-7a-5p & mmu-miR-1953 & $4-5 p$ \\
\hline chr1:132213 & $\mathrm{m}$ & $\begin{array}{l}\text { mmu-miR-7007- } \\
5 p\end{array}$ & $\mathrm{~mm}$ & $\mathrm{mmu}$ & $-3 p$ \\
\hline chrle & $\begin{array}{l}\text { mmu-miR-7660- } \\
3 \mathrm{p}\end{array}$ & $\begin{array}{c}\text { mmu-miR-6992- } \\
3 \mathrm{p}\end{array}$ & mmu-miR-1903 & mmu-miR-6380 & mmu-miR-7665-5p \\
\hline 2 & amu-miR-6339 & mmu-miR-1954 & mmu-miR-6912-5p & amu-miR-500-5p & nmu-miR-7033-5p \\
\hline
\end{tabular}

pathway, and cancer proteoglycan pathway. Our results suggest that the effect of EA on T2DM is multifactorial and is exerted at multiple levels. Regulating the blood sugar level may only be one of the smaller aspects of EA; other therapeutic effects of EA on T2DM still need to be explored.

Interestingly, we found that the effect of EA on the thyroid hormone signaling pathway is very prominent in
T2DM, with both upregulated genes (MED13, MED13L, NCOA2, PIK3CB, and SLC16A10) and downregulated genes (MED12L, PLCG2, PRKCA, and TSC2) being observed. Previous studies have revealed the association of hyperthyroidism [35-37] and hypothyroidism [38-40] with insulin resistance in T2DM. Thyroid hormones could increase the concentration of free fatty acids, thereby 
TABLE 9: The predicted expression of top 10 downregulated circRNA-bound microRNAs affected by EA.

\begin{tabular}{|c|c|c|c|c|c|}
\hline circRNA & miRNA & miRNA & miRNA & miRNA & miRNA \\
\hline chr2:24847944-24863908- & mmu-miR-206-5p & $\begin{array}{l}\text { mmu-miR-7038- } \\
3 p\end{array}$ & mmu-miR-145a-3p & $\begin{array}{l}\text { mmu-miR-7084- } \\
3 p\end{array}$ & mmu-miR-8112 \\
\hline chr19:24346237-24360151- & mmu-miR-1903 & $\begin{array}{l}\text { mmu-miR-6982- } \\
5 p\end{array}$ & mmu-miR-6387 & mmu-miR-107-5p & mmu-miR-3068-5p \\
\hline chr17:24607437-24619570- & mmu-miR-6998-5p & mmu-miR-1906 & mmu-miR-7001-5p & mmu-miR-214-3p & mmu-miR-7222-3p \\
\hline chr11:86532772-86535460- & mmu-miR-29a-5p & $\begin{array}{c}\mathrm{mmu}-\mathrm{miR}-5623- \\
3 \mathrm{p}\end{array}$ & mmu-miR-6395 & mmu-miR-328-3p & mmu-miR-16-1-3p \\
\hline chr2:71875406-71880110+ & mmu-miR-29a-5p & $\begin{array}{l}\text { mmu-miR-6937- } \\
5 \mathrm{p}\end{array}$ & mmu-miR-192-3p & $\begin{array}{l}\text { mmu-miR-7075- } \\
5 \mathrm{p}\end{array}$ & mmu-miR-494-3p \\
\hline chr12:40124567-40128035- & mmu-miR-127-5p & $\begin{array}{c}\text { mmu-miR-3095- } \\
5 \mathrm{p}\end{array}$ & $\begin{array}{l}\text { mmu-miR-6973b- } \\
3 p\end{array}$ & mmu-miR-6365 & mmu-miR-7236-3p \\
\hline chr8:82068932-82071835+ & mmu-miR-7092-3p & $\begin{array}{l}\text { mmu-miR-7090- } \\
3 p\end{array}$ & mmu-miR-761 & $\begin{array}{l}\text { mmu-miR-6934- } \\
5 \mathrm{p}\end{array}$ & mmu-miR-3966 \\
\hline chr8:117555975-117558116+ & mmu-miR-6918-5p & $\begin{array}{l}\text { mmu-miR-7014- } \\
5 \mathrm{p}\end{array}$ & mmu-miR-3079-5p & mmu-miR-212-5p & mmu-miR-432 \\
\hline $\begin{array}{l}\text { chr11:108012627- } \\
108014381-\end{array}$ & mmu-miR-1903 & $\begin{array}{c}\text { mmu-miR-7065- } \\
3 p\end{array}$ & mmu-miR-7215-3p & $\begin{array}{l}\text { mmu-miR-7009- } \\
5 p\end{array}$ & $\begin{array}{c}\text { mmu-miR-3074-2- } \\
3 p\end{array}$ \\
\hline chr3:135655500-135669339- & mmu-miR-145a-5p & mmu-miR-34a-5 & mmu-miR-216a-3 & $\begin{array}{l}\text { mmu-miR-6964- } \\
3 \mathrm{p}\end{array}$ & mmu-miR-3066-5p \\
\hline
\end{tabular}

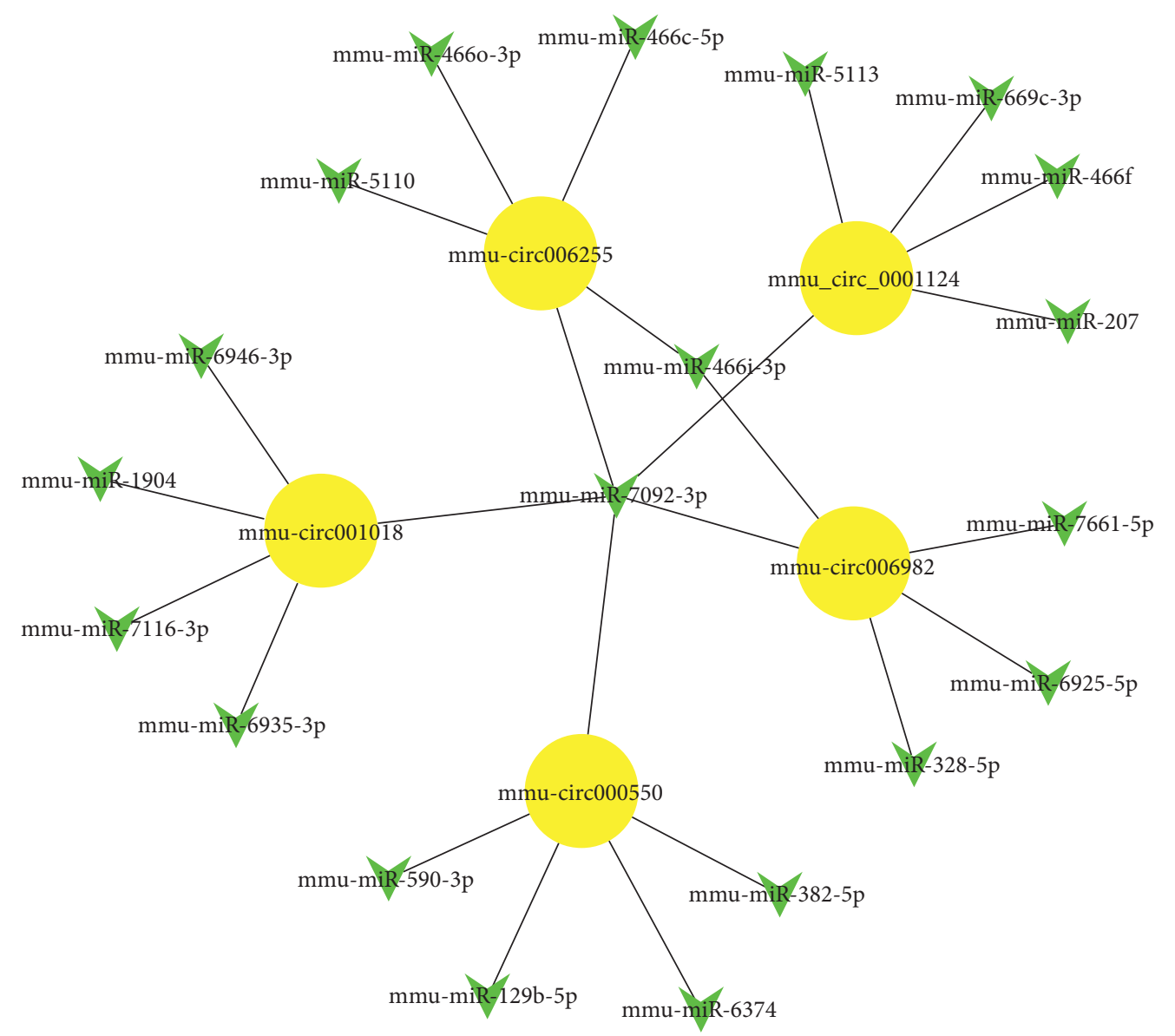

FIGURE 6: The circRNA/miRNA network analysis of two downregulated (yellow nodes) and three upregulated circRNAs (yellow nodes) with their acting miRNAs (green nodes).

enhancing the storage and oxidation of glucose and affecting insulin secretion $[41,42]$. Previously, it was reported that $15 \mathrm{~Hz}$ EA at ST36 improves insulin sensitivity and reduces free fatty acid levels in rats with chronic dexamethasoneinduced insulin resistance [43]. This suggested that EA may improve insulin resistance by regulating thyroid hormones 


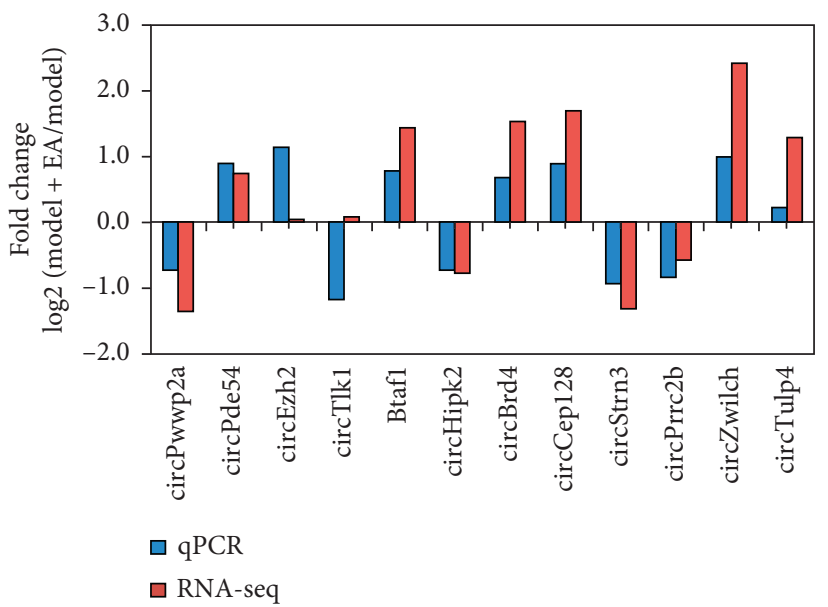

(a)

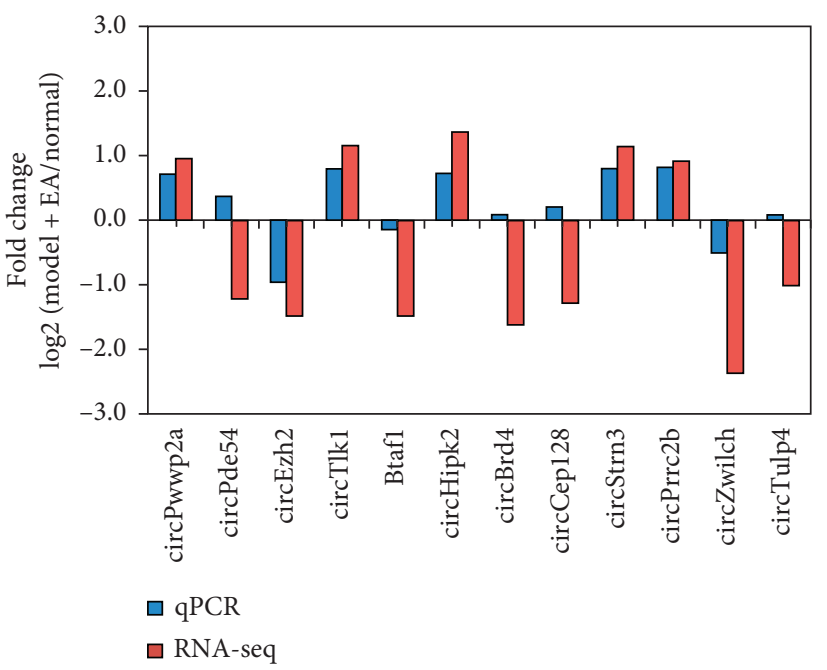

(b)

Figure 7: qRT-PCR validation for the expression of 12 circRNAs. Comparison between RNA-seq data and qPCR results. The vertical axis shows the fold change ( $\log 2$ transformed) of each circRNA between the model + EA and model groups (a) or the model + EA and normal groups measured (b) by qPCR and RNA-seq, respectively.

in T2DM, which needs to be confirmed by further experiments.

In addition, we constructed an interaction network between circRNAs and microRNAs and found that mmumir-7092-3p, one of the most active miRNAs, was closely associated with circINPP4B, which was significantly downregulated by EA. INPP4B is involved in the phosphatidylinositol signaling pathway, which inhibits PI3K/Akt signaling and is emerging as a tumor suppressor in a variety of tissues [44]. The interaction network of circRNA/miRNA further confirmed that the effects of EA on T2DM may involve the PI3K/Akt signaling pathway.

This study has several limitations. First, only 1 replicate has been sequenced from each condition. Second, this study assessed differentially expressed circRNAs via fold changes $\geq 2.0$ or fold change $\leq 0.5$ in the expression level in the model + EA group compared with both the normal and model group. As only 1 replicate per condition was performed, the evaluation criteria of differentially expressed circRNAs cannot contain $P$ value. Third, although the expression levels of selected circRNAs seem to change with EA induction, their specific functions have not been studied or verified. As for the circRNAs function as a sponge, further studies about the effects on the predicted miRNAs or the expression level of the proposed interacting mRNAs are still needed.

\section{Conclusion}

In conclusion, we found that EA intervention can regulate the thyroid hormone and phosphatidylinositol signaling pathway to attenuate apoptosis of islet $\beta$ cells and protect islet function in T2DM mice. This study opens up new avenues for elucidating the mechanism of EA function and provides a reference for finding new therapeutic targets for T2DM.

\section{Data Availability}

The data used to support the findings of this study are included within the article and the supplementary information files. The sequencing data have been uploaded to the Gene Expression Omnibus (GEO) database (GEO ID: GSE133665).

\section{Conflicts of Interest}

The authors declare that there are no conflicts of interest regarding the publication of this paper.

\section{Authors' Contributions}

Yin Shou and Li Hu contributed equally to this work.

\section{Acknowledgments}

We thank Cloudseq Biotech Inc. (Shanghai, China) for highthroughput sequencing service and bioinformatic support. Also, we are grateful to Shanghai Freedom Bio-Tech Co., Ltd., for its help in immunohistochemistry experiments. This work was supported by grants from the National Natural Science Foundation of China (grant nos. 81373755 and 81403470) and Shanghai Science and Technology Commission (grant no. 17401932200).

\section{Supplementary Materials}

Table S1: the primers used in the present study. Table S2: circRNA expression profiling identified in the plasma exosomes of three groups. Table S3: upregulated and downregulated differentially expressed circRNAs among three groups. Table S4: pathways related with upregulated and downregulated host linear transcripts among three groups. Table S5: each circRNA and its potential complementary 
binding miRNAs. Figure S1: plasma exosomes confirmed by western blotting (A), transmission electron microscopy (B), and NTA (C). Figure S1A shows that exosome-enriched markers CD9, TSG101, and CD63 were abundant in plasma exosomes and that no calnexin was observed. Transmission electron microscopy confirmed the shape and morphology of the isolated exosomes. Through NTA, we obtained the total exosome particle number (particles per $\mathrm{ml}$ ). The average exosome number was $8.8 \times 108 \mathrm{ml}$. The vesicle size in the sample was mainly distributed in the range of $70-200 \mathrm{~nm}$, which accorded with the size range of exosomes. The particle size range of $91.6 \%$ was about $152.8 \mathrm{~nm}$. Figure S2 shows the gene ontology (GO) annotation of downregulated host linear transcripts among three groups. Figure S3 shows the gene ontology (GO) annotation of upregulated host linear transcripts among three groups. (Supplementary Materials)

\section{References}

[1] K. Bahartan, K. Horman, A. Gal, A. Drexler, Y. Mayzel, and T. Lin, "Assessing the performance of a noninvasive glucose monitor in People with type 2 diabetes with different demographic profiles," Journal of Diabetes Research, vol. 2017, Article ID 4393497, 8 pages, 2017.

[2] N. H. Cho, J. E. Shaw, S. Karuranga et al., "IDF diabetes atlas: global estimates of diabetes prevalence for 2017 and projections for 2045," Diabetes Research and Clinical Practice, vol. 138, pp. 271-281, 2018.

[3] R. E. Amori, J. Lau, and A. G. Pittas, "Efficacy and safety of incretin therapy in type 2 diabetes," JAMA, vol. 298, no. 2, pp. 194-206, 2007.

[4] Z. Z. Htike, F. Zaccardi, D. Papamargaritis, D. R. Webb, K. Khunti, and M. J. Davies, "Efficacy and safety of glucagonlike peptide- 1 receptor agonists in type 2 diabetes: a systematic review and mixed-treatment comparison analysis," Diabetes, Obesity and Metabolism, vol. 19, no. 4, pp. 524-536, 2017.

[5] M. Li, Y. Yang, D. Jiang, M. Ying, Y. Wang, and R. Zhao, "Efficacy and safety of liraglutide versus sitagliptin both in combination with metformin in patients with type 2 diabetes," Medicine, vol. 96, no. 39, p. e8161, 2017.

[6] L. Zhang, M. Zhang, Y. Zhang, and N. Tong, "Efficacy and safety of dulaglutide in patients with type 2 diabetes: a metaanalysis and systematic review," Scientific Reports, vol. 6, no. 1, p. 18904, 2016.

[7] P. Gæde, P. Vedel, N. Larsen, G. V. H. Jensen, H.-H. Parving, and O. Pedersen, "Multifactorial intervention and cardiovascular disease in patients with type 2 diabetes," New England Journal of Medicine, vol. 348, no. 5, pp. 383-393, 2003.

[8] L. Ji, D. Hu, C. Pan et al., "Primacy of the 3B approach to control risk factors for cardiovascular disease in type 2 diabetes patients," The American Journal of Medicine, vol. 126, no. 10, pp. 925.e11-925.e22, 2013.

[9] P. Zeitler, K. Hirst, L. Pyle et al., "A clinical trial to maintain glycemic control in youth with type 2 diabetes," New England Journal of Medicine, vol. 366, no. 24, pp. 2247-2256, 2012.

[10] C.-H. Hsu, K.-C. Hwang, C.-L. Chao, J.-G. Lin, S.-T. Kao, and P. Chou, "Effects of electroacupuncture in reducing weight and waist circumference in obese women: a randomized crossover trial," International Journal of Obesity, vol. 29, no. 11, pp. 1379-1384, 2005.

[11] J. M. Lacey, A. M. Tershakovec, and G. D. Foster, “Acupuncture for the treatment of obesity: a review of the evidence," International Journal of Obesity, vol. 27, no. 4, pp. 419-427, 2003.

[12] A. J. M. Boulton, R. A. Malik, J. C. Arezzo, and J. M. Sosenko, "Diabetic somatic neuropathies," Diabetes Care, vol. 27, no. 6, pp. 1458-1486, 2004.

[13] A. J. M. Boulton, A. I. Vinik, J. C. Arezzo et al., "Diabetic neuropathies: a statement by the American diabetes association," Diabetes Care, vol. 28, no. 4, pp. 956-962, 2005.

[14] C.-P. Wang, C.-H. Kao, W.-K. Chen, W.-Y. Lo, and C.-L. Hsieh, "A single-blinded, randomized pilot study evaluating effects of electroacupuncture in diabetic patients with symptoms suggestive of gastroparesis," The Journal of Alternative and Complementary Medicine, vol. 14, no. 7, pp. 833-839, 2008.

[15] Y. J. Kim, "Acupuncture for management of type 2 diabetes mellitus in a patient with myasthenia gravis: a case report," Journal of Acupuncture and Meridian Studies, vol. 10, no. 4, pp. 290-293, 2017.

[16] Z. Qi, Y. Pang, L. Lin et al., "Acupuncture combined with hydrotherapy in diabetes patients with mild lower-extremity arterial disease: a prospective, randomized, nonblinded clinical study," Medical Science Monitor, vol. 24, pp. 28872900, 2018.

[17] J. Cornejo-Garrido, F. Becerril-Chávez, G. Carlín-Vargas et al., "Antihyperglycaemic effect of laser acupuncture treatment at BL20 in diabetic rats," Acupuncture in Medicine, vol. 32, no. 6, pp. 486-494, 2014.

[18] P. V. Peplow and G. D. Baxter, "Electroacupuncture for control of blood glucose in diabetes: literature review," Journal of Acupuncture and Meridian Studies, vol. 5, no. 1, pp. 1-10, 2012.

[19] D. Lan, N. Xu, J. Sun et al., "Electroacupuncture mitigates endothelial dysfunction via effects on the Pi3K/Akt signalling pathway in high fat diet-induced insulin-resistant rats," Acupuncture in Medicine, vol. 36, no. 3, pp. 162-169, 2018.

[20] J. Leng, F. Xiong, J. Yao et al., "Electroacupuncture reduces weight in diet-induced obese rats via hypothalamic Tsc1 promoter demethylation and inhibition of the activity of mTORC1 signaling pathway," Evidence-Based Complementary and Alternative Medicine, vol. 2018, Article ID 3039783, 10 pages, 2018.

[21] A. Tominaga, N. Ishizaki, Y. Naruse, H. Kitakoji, and Y. Yamamura, "Repeated application of low-frequency electroacupuncture improves high-fructose diet-induced insulin resistance in rats," Acupuncture in Medicine, vol. 29, no. 4, pp. 276-283, 2011.

[22] S. M. van Dommelen, P. Vader, S. Lakhal et al., "Microvesicles and exosomes: opportunities for cell-derived membrane vesicles in drug delivery," Journal of Controlled Release, vol. 161, no. 2, pp. 635-644, 2012.

[23] S.-J. Zhang, X. Chen, C.-P. Li et al., "Identification and characterization of circular RNAs as a new class of putative biomarkers in diabetes retinopathy," Investigative Opthalmology \& Visual Science, vol. 58, no. 14, pp. 65006509, 2017.

[24] K. Shan, C. Liu, B.-H. Liu et al., "Circular noncoding RNA HIPK3 mediates retinal vascular dysfunction in diabetes mellitus," Circulation, vol. 136, no. 17, pp. 1629-1642, 2017.

[25] G. Jiang, Y. Ma, T. An et al., "Relationships of circular RNA with diabetes and depression," Scientific Reports, vol. 7, no. 1, p. 7285, 2017.

[26] W. Sisi, L. I. Jiajianghui, D. Jing, and Y. Yuxiang, "Screening and validation of type 2 diabetes mellitus related circRNAs," Chinese Journal of Diabetes, vol. 2, pp. 86-90, 2019. 
[27] Y. Wu, B. Li, and X.-M. Gao, "Selective fluorescence of zymogen granules of pancreatic acinar cells stained with hematoxylin and eosin," Biotechnic \& Histochemistry, vol. 77, no. 5-6, pp. 291-293, 2002.

[28] Z. Ao, M. Meloche, T. Kieffer, G. Warnock, and L. Marzban, "The glucagon-like peptide-1 analogue exenatide reduces beta-cell apoptosis and enhances survival of cultured human islets: implications for clinical islet transplantation," Canadian Journal of Diabetes, vol. 32, no. 4, p. 337, 2008.

[29] P. Ly, L. S. Teitz, D. H. Kim et al., "Selective Y centromere inactivation triggers chromosome shattering in micronuclei and repair by non-homologous end joining," Nature Cell Biology, vol. 19, no. 1, pp. 68-75, 2017.

[30] A. Dobin, C. A. Davis, F. Schlesinger et al., "STAR: ultrafast universal RNA-seq aligner," Bioinformatics, vol. 29, no. 1, pp. 15-21, 2013.

[31] T. Jakobi, L. F. Czaja-Hasse, R. Reinhardt, and C. Dieterich, "Profiling and validation of the circular RNA repertoire in adult murine hearts," Genomics, Proteomics \& Bioinformatics, vol. 14, no. 4, pp. 216-223, 2016.

[32] H. B. Ma, Y. N. Yao, J. J. Yu, X. X. Chen, and H. F. Li, "Extensive profiling of circular RNAs and the potential regulatory role of circRNA-000284 in cell proliferation and invasion of cervical cancer via sponging miR-506," American Journal of Translational Research, vol. 10, no. 2, pp. 592-604, 2018.

[33] B.-Y. Cao, R. Li, H.-H. Tian et al., "PI3K-GLUT4 signal pathway associated with effects of EX-B3 electroacupuncture on hyperglycemia and insulin resistance of T2DM rats," Evidence-Based Complementary and Alternative Medicine, vol. 2016, Article ID 7914387, 10 pages, 2016.

[34] Y. L. Jiang, Y. Ning, Y. Y. Liu et al., "Effects of preventive acupuncture on streptozotocin-induced hyperglycemia in rats," Journal of Endocrinological Investigation, vol. 34, no. 10, pp. e355-e361, 2011.

[35] M. Centeno Maxzud, L. Gomez Rasjido, M. Fregenal et al., "Prevalence of thyroid dysfunction in patients with type 2 diabetes mellitus," Medicina, vol. 76, no. 6, pp. 355-358, 2016.

[36] L.-H. Meng, Y. Huang, J. Zhou et al., "Use of first-phase insulin secretion in early diagnosis of thyroid diabetes and type 2 diabetes mellitus," Chinese Medical Journal, vol. 130, no. 7, pp. 798-804, 2017.

[37] I. Subekti, L. A. Pramono, E. Dewiasty, and D. S. Harbuwono, "Thyroid dysfunction in type 2 diabetes mellitus patients," Acta medica Indonesiana, vol. 49, no. 4, pp. 314-323, 2017.

[38] B. Ujwal Upadya, M. N. Suma, K. M. Srinath, P. Akila, D. Parveen, and S. V. Shilpa, "Effect of insulin resistance in assessing the clinical outcome of clinical and subclinical hypothyroid patients," Journal of Clinical and Diagnostic Research, vol. 9, no. 2, pp. OC01-OC04, 2015.

[39] E. Maratou, D. J. Hadjidakis, A. Kollias et al., "Studies of insulin resistance in patients with clinical and subclinical hypothyroidism," European Journal of Endocrinology, vol. 160, no. 5, pp. 785-790, 2009.

[40] N. Yang, Z. Yao, L. Miao et al., "Novel clinical evidence of an association between homocysteine and insulin resistance in patients with hypothyroidism or subclinical hypothyroidism," PLoS One, vol. 10, no. 5, Article ID e0125922, 2015.

[41] A. Prats-Puig, C. Sitjar, R. Ribot et al., "Relative hypoadiponectinemia, insulin resistance, and increased visceral fat in euthyroid prepubertal girls with low-normal serum free thyroxin," Obesity, vol. 20, no. 7, pp. 1455-1461, 2012.

[42] L. van Tienhoven-Wind, G. Dallinga-Thie, and R. Dullaart, "Higher plasma ApoE levels are associated with low-normal thyroid function: studies in diabetic and nondiabetic subjects," Hormone and Metabolic Research, vol. 48, no. 7, pp. 462-467, 2016.

[43] C.-Y. Tzeng, Y.-C. Lee, J.-J. Chung et al., "15 Hz electroacupuncture at St 36 improves insulin sensitivity and reduces free fatty acid levels in rats with chronic dexamethasoneinduced insulin resistance," Acupuncture in Medicine, vol. 34, no. 4, pp. 296-301, 2016.

[44] C. C. Jiang, M. N. Chi, S. T. Guo et al., “Abstract 4718: Inositol polyphosphate 4-phosphatase II activates PI3K/SGK3 signaling to promote proliferation of human melanoma cells," in Proceedings of the AACR 106th Annual Meeting 2015, April 2015. 


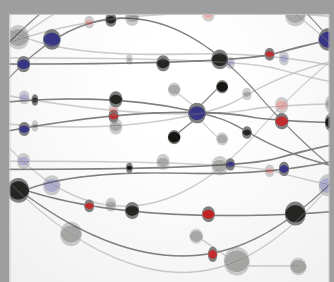

The Scientific World Journal
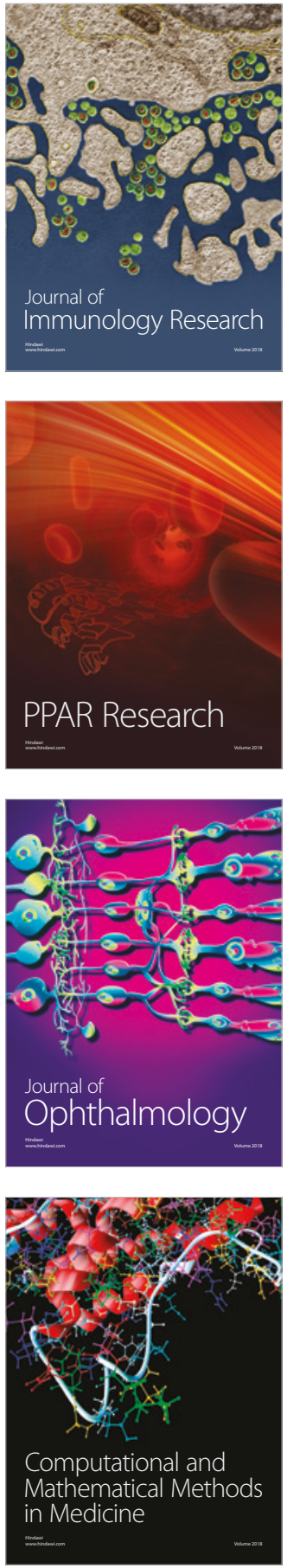

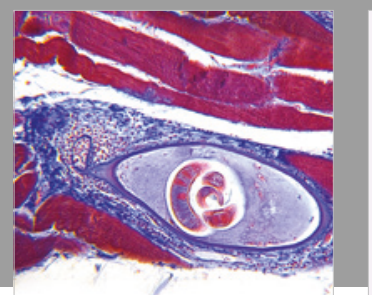

Gastroenterology Research and Practice

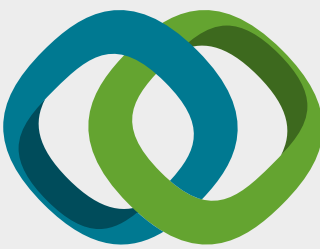

\section{Hindawi}

Submit your manuscripts at

www.hindawi.com
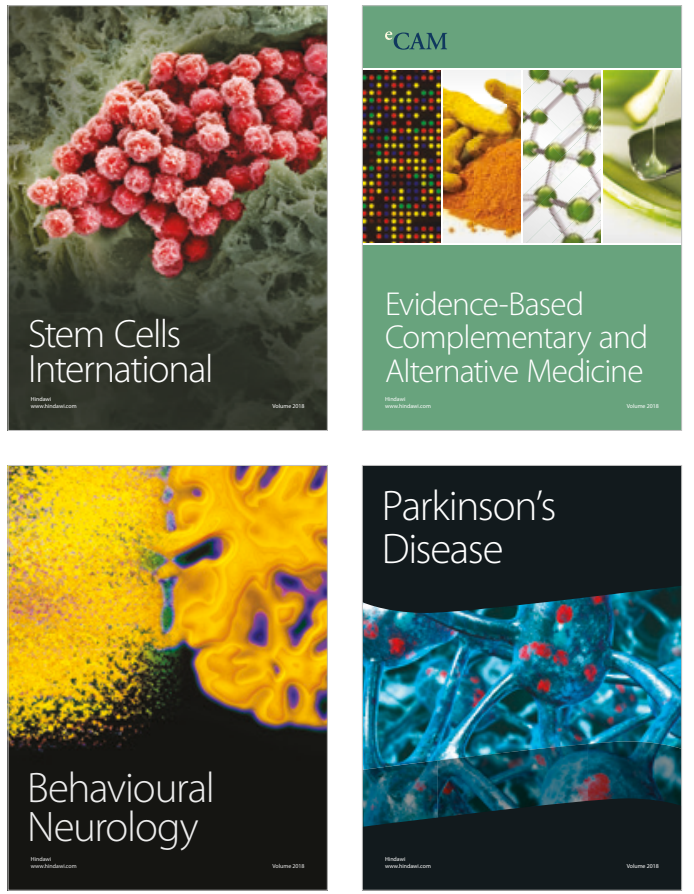

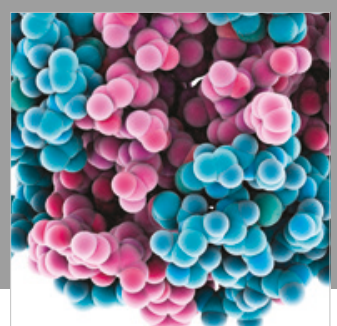

ournal of

Diabetes Research

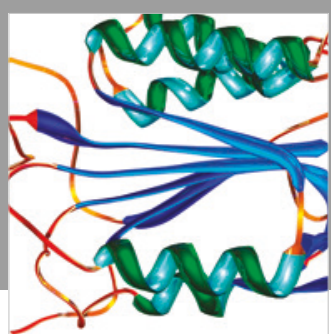

Disease Markers
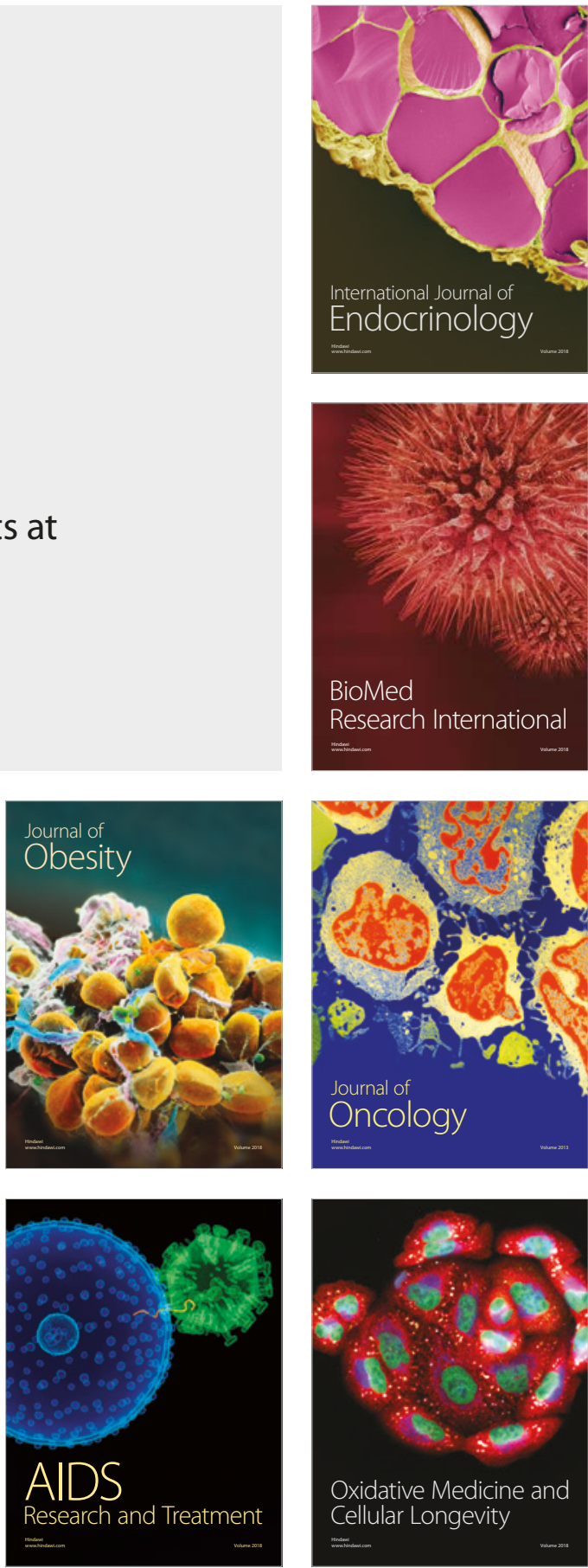